\title{
A Radio-Frequency Sheath Boundary Condition and its Effect on Slow Wave Propagation
}

\author{
D. A. D'Ippolito and J. R. Myra \\ Lodestar Research Corporation, 2400 Central Avenue, Boulder, Colorado 80301
}

May, 2006; revised September, 2006

(to be published in Physics of Plasmas) 


\title{
A radio-frequency sheath boundary condition and its effect on slow wave propagation*
}

\author{
D. A. D'Ippolito ${ }^{\dagger}$ and J. R. Myra \\ Lodestar Research Corporation, 2400 Central Avenue, Boulder, Colorado 80301
}

\begin{abstract}
Predictive modeling of radiofrequency wave propagation in high-power fusion experiments requires accounting for nonlinear losses of wave energy in the plasma edge and at the wall. An important mechanism of "anomalous" power losses is the acceleration of ions into the walls by rf sheath potentials. Previous work computed the "sheath power dissipation" non-self-consistently by post-processing fields obtained as the solution of models which did not retain sheaths. Here, a method is proposed for a self-consistent quantitative calculation of sheath losses by incorporating a sheath boundary condition (SBC) in antenna coupling and wave propagation codes. It obtains the self-consistent sheath potentials and spatial distribution of the time-averaged power loss in the solution for the linear rf fields. It can be applied for ion cyclotron and (in some cases) lower hybrid waves. The use of the SBC is illustrated by applying it to the problem of an electron plasma wave propagating in a waveguide. This model problem is relevant to understanding the low heating efficiency in direct ion-Bernstein wave launch. Implications for calculating sheath voltages driven by fast-wave antennas are also discussed.
\end{abstract}

PACS numbers: 52.35.Mw, 52.40.Fd, 52.40.Kh, 52.50.Qt, 52.55.Fa

†email: dippolito@lodestar.com 


\section{Introduction}

A great deal of research and computational effort in the fusion program has been devoted to the linear theory of rf wave propagation in the ion-cyclotron range of frequencies (ICRF), and this effort has been very successful in understanding many aspects of ICRF heating and current drive experiments. However, a growing body of experimental and theoretical work has shown that nonlinear effects also play an important role in coupling high power rf waves to plasmas. (The reader is referred to Ref. 1 for a detailed review of nonlinear rf physics relevant to fusion experiments and an extensive list of references.) One of the key concepts is that an rf electric field component $E_{\|}$ parallel to the equilibrium magnetic field $\mathbf{B}$ will, in order to confine electrons, generate an rf sheath potential of order $\mathrm{V}=\int \mathrm{ds} \mathrm{E}_{\|}$(integrated along $\mathbf{B}$ ). ${ }^{2-6}$ In order to satisfy Maxwell's equations in the vicinity of a metal boundary, most of the rf voltage drop V must occur within the sheaths, as discussed in Sec. II of this paper, so that the rf field distribution along the field line in the presence of the sheaths is different than that computed using vacuum rf fields. This oscillating sheath potential is "rectified" by the requirement of plasma ambipolarity to produce a time-independent (dc) plasma bias potential. For megawatt power coupling, the rectified potential is generally large $(\sim \mathrm{kV})$ and has important consequences. In fusion experiments, rf sheaths cause a variety of deleterious interactions, such as impurity generation, ${ }^{7}$ convective transport, ${ }^{8-10}$ hot spots $^{11,12}$ and damage to the antenna and surrounding structures, and power dissipation, ${ }^{13}$ caused by both the near-field 7 and far-field ${ }^{14}$ waves. In many experiments, the heating efficiency with low- $\mathrm{k}_{\|}$waves is poor, and sheath power dissipation is a likely candidate to explain this phenomenon.

Even in present short-pulse experiments, these nonlinear effects can impose limitations, e.g. by restricting operation to high- $\mathrm{k}_{\|}$phasing 7 or lower power levels. In longer-pulse experiments ${ }^{15}$ and future burning plasma experiments, the requirements will 
be even more severe: even a small level of antenna-plasma interaction may result damaging localized heat deposition during the shot.

While the work cited here and in Ref. 1 has elucidated the physics of many of the important nonlinear rf interactions, what is lacking is the ability to make self-consistent, quantitative predictions. Such calculations are necessary for interpreting experiments, designing improved antennas, and evaluating operating scenarios for the next generation of fusion experiments. The present paper takes a step in this direction by demonstrating a procedure for self-consistent rf sheath calculations.

The goal of this paper is two-fold. First, we describe a sheath boundary condition (SBC) which can be used in linear rf wave propagation and antenna coupling codes to calculate the sheath voltage and sheath power dissipation self-consistently. This boundary condition (BC) is an extension of one presented earlier, ${ }^{14}$ and was briefly described in a recent conference paper. ${ }^{16}$ Second, we demonstrate the use of the SBC in an analytic calculation of electron plasma wave (EPW) propagation down a plasma-filled metallic waveguide. The EPW calculation demonstrates the modification of the rf wave spatial structure by the sheaths and wave decay due to sheath power dissipation. Energy conservation is verified for this model. The calculation is relevant to direct-launch ion Bernstein wave (IBW) experiments using the mode-transformation (EPW to IBW) technique, and supports earlier calculations 17,18 which suggested that sheath power dissipation made it difficult to couple IBW power to the core plasma by direct launch.

Note that slow wave (SW) launchers such as IBW antennas produce propagating $\mathrm{E}_{\|}$fields and thus create large sheath potentials $(\sim 1 \mathrm{keV})$ on nearby material surfaces, but even fast wave (FW) antennas can create substantial near-field ${ }^{3-7}$ and far-field ${ }^{14}$ sheaths by coupling to unwanted SWs. There is some experimental evidence that FW sheaths dissipate significant power in low- $\mathrm{k}_{\|}$phasing, contributing to reduced heating efficiency, ${ }^{19,20}$ but until now we have not a way of calculating the lost power. The sheath 
BC described here is relevant for both SW and FW codes, and it provides a unified way of describing both near- and far-field sheaths.

It should be noted that the problem addressed here occurs in a variety of other contexts as well, including lower-hybrid heating in fusion plasmas, rf plasma sources for particle accelerators, plasma-filled waveguides, and plasma processing devices. In particular, there is a vast literature on sheath modeling in low temperature plasmas and plasma processing. The present work thus has potentially broad applicability in plasma

devices. Our proposed sheath BC is compatible with sheath models ${ }^{21-24}$ used in plasma processing and can also be applied to lower hybrid (LH) frequency waves in some cases. The sheath theory used here assumes inertia-free electrons and thus breaks down at sufficiently high frequency, as discussed in Sec. II D and Appendix B.

The plan of this paper is as follows. The general rf sheath BC is described in Sec. II. The EPW model problem is described and solved in Sec. III. This calculation illustrates the basic physics of sheath power dissipation by ion acceleration and the redistribution of the self-consistent rf electric fields between the main plasma and sheath regions. Although the sheath $\mathrm{BC}$ is applicable to electromagnetic wave propagation, the EPW illustration is purely electrostatic, and thus exhibits some differences from the electromagnetic antenna coupling problem. This issue is discussed in Sec. IV after summarizing the main results. The proof of energy conservation is given in Appendix A to verify that the formalism is self-consistent. Appendix B discusses the high-frequency limit of the SBC and the EPW model problem to elucidate the conditions under which the theory applies to LH waves.

\section{Rf Sheath BC}

Sheaths of the type considered here (i.e. with a not-too-small component of magnetic field directed into the surface) are depleted of electrons to ensure ambipolarity. 
The theory is valid in the frequency regime $\omega_{\mathrm{pi}} \sin \alpha<\omega<\omega_{\mathrm{pe}} \sin \alpha$ (i.e. immobile ions and inertia-free electrons) and for equilibrium magnetic fields satisfying $\sin \alpha=B_{n} / B>$ $\left(m_{e} / m_{i}\right)^{1 / 2}$, where $B_{n}$ is the component of $\mathbf{B}$ normal to the sheath and $\omega_{p j}$ is the plasma frequency for species $\mathrm{j}$.

The main effect of the sheaths on the rf wave (our interest here) is to eliminate the normally large parallel electron plasma response in the sheath region. Thus to lowest order, an rf wave sees a sheath as a vacuum layer. For reasons that will become evident, here we treat the sheath as an insulating dielectric medium with scalar dielectric constant

$$
\varepsilon_{\text {sh }}=1+\mathrm{i} v
$$

and assume that the sheath impedance is dominated by its capacitance $(v<<1)$. In Sec. IIA, we derive the modified BC for the rf electric field $\mathbf{E}$, which is a simple consequence of Maxwell's equations, and in Sec. IIB show how to incorporate the sheath power dissipation (or other dissipative mechanisms) into the calculation. A demonstration of energy conservation for the model problem is given in Appendix A.

\section{A. Sheath Capacitance and rf Field Modification}

It is useful to write the rf electric field $\mathbf{E}$ relative to both the sheath surface and the local magnetic field $\mathbf{B}$

$$
\mathbf{E}=\left(E_{n} \mathbf{n}+\mathbf{E}_{t}\right) \mathrm{e}^{\mathrm{i} \theta}=\left(E_{\|} \mathbf{b}+\mathbf{E}_{\perp}\right) \mathrm{e}^{\mathrm{i} \theta},
$$

where $\theta=(\mathbf{k} \cdot \mathbf{x}-\omega t), \mathbf{n}$ is the unit vector normal to the sheath (pointing into the plasma), and $\mathbf{b}=\mathbf{B} / \mathbf{B}$ is the unit vector along the B-field direction. For definiteness, in this paper we take $\mathrm{z}$ as the direction normal to the sheath $\left(\mathbf{n}=\hat{\mathbf{e}}_{\mathrm{z}}\right)$. Here, the subscript $\mathrm{t}$ denotes the component tangential to the sheath surface, and the subscript $\perp$ denotes the component perpendicular to the magnetic field line. This geometry is shown in Fig. 1.

The condition for sheaths to develop is that electrons have a sufficiently large component of their motion into the bounding surface, which requires 


$$
\mathbf{n} \cdot \mathbf{b} \equiv \sin \alpha>\left(\mathrm{m}_{\mathrm{e}} / \mathrm{m}_{\mathrm{i}}\right)^{1 / 2}
$$

We also assume that the sheath is "thin" so that the electrostatic (ES) approximation is valid in the sheath region. Denoting the time-averaged sheath width by $\Delta$, this requires

$$
\left|\mathbf{k}_{\mathrm{t}}\right| \Delta<<1,
$$

which is typically valid. In this limit, the $\operatorname{rf}$ sheath potential $\Phi \propto z$, and $E_{n}(z)=-\nabla_{z} \Phi$ is constant to lowest order in $\mathrm{k}_{\mathrm{t}} \Delta$, implying $\nabla^{2} \Phi=0$. We are interested here in the effect of the sheaths on the wave component at frequency $\omega$ (and on the dc power dissipation); thus, we can neglect the oscillatory motion of the sheath at frequency $\omega$ (which generates higher harmonics 21,22 ) and apply the $\mathrm{BC}$ at the location of the time-averaged sheath width.

The derivation of the SBC begins by imposing continuity of $D_{n}=\mathbf{n} \cdot \mathbf{D}=\mathbf{n} \cdot \boldsymbol{\varepsilon} \cdot \mathbf{E}$

$$
\mathrm{D}_{\mathrm{n}}^{(\mathrm{sh})} \equiv \varepsilon_{\mathrm{sh}} \mathrm{E}_{\mathrm{n}}^{(\mathrm{sh})}=\mathrm{D}_{\mathrm{n}}^{(\mathrm{pl})}
$$

where the superscripts (pl) and (sh) denote quantities on the plasma and sheath sides of the sheath-plasma boundary, respectively, and $\varepsilon_{\mathrm{sh}}$ is the scalar defined in Eq. (1). (For tensor $\boldsymbol{\varepsilon}$, the component $\mathbf{n} \cdot \boldsymbol{\varepsilon} \cdot \mathbf{n}$ would be used here.) In the ES limit, we have

$$
-\varepsilon_{\mathrm{sh}} \nabla_{\mathrm{n}} \Phi=\mathrm{D}_{\mathrm{n}}^{(\mathrm{pl})}
$$

and integrating from the metal wall through the sheath in the direction of $\mathbf{n}$ yields

$$
-\Phi^{(\mathrm{sh})}=\frac{\Delta}{\varepsilon_{\mathrm{sh}}} \mathrm{D}_{\mathrm{n}}^{(\mathrm{pl})}
$$

where $\Phi^{(\mathrm{sh})}=0$ in the metal and $\Phi=\Phi^{(\mathrm{sh})}$ at the sheath-plasma interface. Taking the tangential gradient of Eq. (7) with $\mathbf{E}_{\mathrm{t}}=-\mathrm{i} \mathrm{k}_{\mathrm{t}} \Phi$ yields

$$
\mathrm{E}_{\mathrm{t}}^{(\mathrm{sh})}=\nabla_{\mathrm{t}}\left(\frac{\Delta}{\varepsilon_{\mathrm{sh}}} \mathrm{D}_{\mathrm{n}}^{(\mathrm{pl})}\right) .
$$


Using the continuity of $\mathbf{E}_{\mathrm{t}}$ across the sheath-plasma interface, we obtain the final sheath $\mathrm{BC}$ on the rf fields on the plasma side

$$
\mathbf{E}_{\mathrm{t}}^{(\mathrm{pl})}=\nabla_{\mathrm{t}}\left(\frac{\Delta}{\varepsilon_{\mathrm{sh}}} \mathrm{D}_{\mathrm{n}}^{(\mathrm{pl})}\right)
$$

which is a constraint involving only the rf fields on the plasma side of the sheath-plasma boundary. Equation (9) is the main result of this section.

The form of the SBC presented here is valid for both frequency-domain 16 and time-domain ${ }^{25}$ codes. The SBC in Eq. (9) is the electrostatic, thin-sheath limit of a fully electromagnetic BC described in the Appendix of Ref. 14. It is also closely related to BCs being tested in simulations of plasma processing. ${ }^{23,24}$

In the limit where the sheath width vanishes $(\Delta \rightarrow 0)$, Eq. (9) reduces to the usual BC at a conducting surface: $\mathbf{E}_{\mathrm{t}}^{(\mathrm{pl})}=0$. The extra term in Eq. (9) represents the effect of the sheath capacitance ( $\propto \varepsilon_{\mathrm{sh}} / \Delta$ for the case of $\varepsilon_{\mathrm{sh}}$ nearly real and positive) and results from the physical approximation that the plasma current is carried across the sheath by the displacement current. This requires that the rf current carried by ions across the sheath is negligible and is satisfied in the "immobile ion" limit, $\omega>\omega_{\mathrm{pi}} \sin \alpha$.

The sheath BC can be extended to include multiple layers. For example, consider the case where the metal is coated by a thin layer of insulating material, which in turn is in contact with a plasma sheath. Then Eq. (7) is easily generalized (in a slightly different notation) to

$$
\Delta \Phi=\Delta \Phi_{\mathrm{ins}}+\Delta \Phi_{\mathrm{sh}}=-\mathrm{D}_{\mathrm{z}}\left(\frac{\Delta \mathrm{z}_{\mathrm{ins}}}{\varepsilon_{\mathrm{zz}, \mathrm{ins}}}+\frac{\Delta \mathrm{z}_{\mathrm{sh}}}{\varepsilon_{\mathrm{zz}, \mathrm{sh}}}\right), \Delta \Phi_{\mathrm{sh}}=-\mathrm{D}_{\mathrm{z}} \frac{\Delta \mathrm{z}_{\mathrm{sh}}}{\varepsilon_{\mathrm{zz}, \mathrm{sh}}},
$$

where the subscripts "ins" and "sh" refer to the insulating and sheath regions, $\Delta \mathrm{z}_{\mathrm{j}}$ is the width of the jth layer, $\varepsilon_{\mathrm{zz}}$ is the normal component of the dielectric tensor, and $\mathrm{D}_{\mathrm{z}}$ is the normal component of $\mathbf{D}$ (which is constant across all regions). This result shows how the total voltage across the system, $\Delta \Phi=\int \mathrm{dz} \mathrm{E}_{\mathrm{z}}$, is divided between the sheath $\left(\Delta \Phi_{\mathrm{sh}}\right)$ and 
the insulator $\left(\Delta \Phi_{\text {ins }}\right)$, depending on the insulator thickness and dielectric constant. It has been shown experimentally that insulating (boron nitride) coatings on antenna limiters can reduce the rf sheath voltages $\left(\Delta \Phi_{\mathrm{sh}} / \Delta \Phi<<1\right)$ in ICRF experiments. ${ }^{26-28}$ Taking the tangential gradient $\nabla_{\mathrm{t}}$ of Eq. (10), one obtains a BC that can be implemented in rf codes to obtain quantitative estimates of the sheath-reduction effect of insulating limiters.

\section{B. Sheath Resistance and Power Dissipation}

The discussion up to this point has not taken into account energy losses in the sheaths. The sheath power dissipation can be included by substituting Eq. (1) with $v \neq 0$ into the expression for the plasma current

$$
J_{z}=\sigma_{z z} E_{z}=-i\left(\varepsilon_{z z}^{(s h)}-1\right) \frac{\omega}{4 \pi} E_{z}=\frac{\omega v}{4 \pi} E_{z}
$$

and using Eq. (11) in the expression for the total power dissipated in a dielectric medium

$$
\begin{aligned}
& \mathrm{P}_{\text {tot }} \equiv \int \mathrm{dV} \frac{1}{4}\left(\mathbf{J} \cdot \mathbf{E}^{*}+\mathrm{cc}\right), \\
& \quad=\frac{1}{2} \operatorname{Re}\left(\sigma_{\mathrm{zz}}\right)\left|\mathrm{E}_{\mathrm{z}}\right|^{2} \mathrm{~A}_{\mathrm{t}} \Delta=\frac{\omega \mathrm{V}}{8 \pi}\left|\mathrm{E}_{\mathrm{z}}\right|^{2} \mathrm{~A}_{\mathrm{t}} \Delta .
\end{aligned}
$$

Here, $A_{t}$ is the area of the sheaths tangential to the surface, and the sheath width $\Delta$ is assumed to satisfy the well-known Child-Langmuir Law

$$
\Delta=\lambda_{\mathrm{D}}\left(\frac{\mathrm{eV}_{0}}{\mathrm{~T}_{\mathrm{e}}}\right)^{3 / 4},
$$

where $\lambda_{\mathrm{D}}=\left(\mathrm{T}_{\mathrm{e}} / 4 \pi \mathrm{ne}^{2}\right)^{1 / 2} \approx \mathrm{v}_{\text {the }} / \omega_{\mathrm{pe}}$ is the electron Debye length, and $\mathrm{V}_{0}$ is the rectified (dc) sheath potential which ensures that the time-averaged current to the sheaths vanishes (see also Sec. II D). Various (similar) models ${ }^{13,21,22}$ have been derived for $\mathrm{V}_{0}$ giving the transition between dc Bohm sheaths and rf sheaths with $\mathrm{eV}_{\mathrm{sh}} / \mathrm{T}_{\mathrm{e}}>>1$, but for conceptual simplicity a useful approximate expression is

$$
\mathrm{V}_{0} \approx \mathrm{C}_{\mathrm{sh}} \mathrm{V}_{\mathrm{sh}}+\mathrm{V}_{\mathrm{B}}
$$


Here $\mathrm{C}_{\mathrm{sh}}$ is an order-unity constant giving the "rectification factor" $6,21,22$ for rf sheaths, $\mathrm{V}_{\mathrm{sh}}$ is the instantaneous sheath voltage (defined below), and $\mathrm{V}_{\mathrm{B}}$ is the Bohm sheath potential given by

$$
\mathrm{V}_{\mathrm{B}}=\frac{\mathrm{T}_{\mathrm{e}}}{\mathrm{e}} \ln \left(\left(\frac{\mathrm{m}_{\mathrm{i}}}{\mathrm{m}_{\mathrm{e}}}\right)^{1 / 2} \sin \alpha\right)
$$

The preceding expression for $\mathrm{V}_{0}$ is correct in the limits $\xi=\mathrm{Ze}\left|\mathrm{V}_{\mathrm{sh}}\right| / \mathrm{T}_{\mathrm{e}}<<1$ and $\xi>>1$ and provides a smooth interpolation for intermediate cases. The Bohm sheath potential must be included in $\mathrm{V}_{0}$ to ensure that $\Delta \sim \lambda_{\mathrm{D}}$ when the rf fields are small.

In the present work, Eq. (12) is interpreted phenomenologically and can be used to include any power loss mechanisms associated with the sheaths (or the sum of all such mechanisms). However, in the present paper we equate $\mathrm{P}_{\text {tot }}$ with the power lost by ions accelerated in the dc sheath potential, viz.

$$
\mathrm{P}_{\mathrm{sh}} \equiv \mathrm{Zn}_{\mathrm{e}} \mathrm{C}_{\mathrm{s}} \mathrm{T}_{\mathrm{e}} \xi \mathrm{h}(\xi) \frac{\mathrm{I}_{1}(\xi)}{\mathrm{I}_{0}(\xi)} \mathrm{A}_{\perp} \rightarrow \mathrm{C}_{\mathrm{sh}} \mathrm{n}_{\mathrm{i}} \mathrm{C}_{\mathrm{s}} \mathrm{ZeV}_{\mathrm{sh}} \mathrm{A}_{\perp}
$$

where $A_{\perp}=|\mathbf{n} \cdot \mathbf{b}| A_{t}$ is the area of the sheaths normal to $\mathbf{B}$. The first expression for $\mathrm{P}_{\text {sh }}$ was derived in Ref. 13 for modeling the transition from the weak to the strong rf regime; the second expression in Eq. (16) gives the $\xi>>1$ limit. Here, $h(\xi)=\left(0.5+C_{\text {sh }} \xi\right) /(1+\xi)$ is a form factor connecting known results in the $\xi<<1$ and $\xi>>1$ limits and $\mathrm{C}_{\mathrm{sh}} \approx 0.6$ (for 0-to-peak voltages). The assumptions under which the expression for $\mathrm{P}_{\text {sh }}$ is compatible with a local sheath BC are discussed further in Sec. II D.

Sheath power loss mechanisms that have been considered by other authors (e.g. in the context of plasma processing) but are omitted here include: joule heating losses in collisional rf sheaths, and Fermi acceleration of electrons transiting between two oscillating rf sheaths at opposite ends of a field line. For any such mechanism, the phase shift ( $\propto v)$ between the rf current and the rf field may not be exact in the present model, but it should give a reasonable estimate of the total sheath power dissipation in the limit $v$ 
$<<1$, as demonstrated explicitly by the example in Sec. III and the analysis in the Appendix.

The following definition of the absolute value of the instantaneous rf sheath voltage completes the specification of the $\mathrm{BC}$

$$
\mathrm{V}_{\mathrm{sh}} \equiv\left|\int \mathrm{dz} \mathrm{E}_{\mathrm{n}}\right| \approx \Delta\left|\mathrm{E}_{\mathrm{n}}\right|
$$

where the integral is taken across the sheath in the direction normal to the sheath boundary. These equations can be solved to obtain an expression for $v$

$$
v=\frac{8 \pi \mathrm{P}_{\mathrm{sh}}}{\omega \mathrm{A}_{\mathrm{t}} \Delta\left|\mathrm{E}_{\|}\right|^{2}}=\frac{8 \pi \Delta\left(\mathrm{P}_{\mathrm{sh}} / \mathrm{A}_{\mathrm{t}}\right)}{\omega \mathrm{V}_{\mathrm{sh}}{ }^{2}}
$$

\section{Implementation}

The nonlinear relationships imposed by sheath physics on $\mathrm{E}_{\mathrm{t}}, \mathrm{D}_{\mathrm{n}}, \Delta, \mathrm{V}_{\mathrm{sh}}$ and $\mathrm{P}_{\mathrm{sh}}$ are expressed by Eqs. (1), (9), (13) - (18). This set of equations constitutes the complete sheath BC, including both the sheath capacitance and resistance. Note that Eqs. (13) and (17) imply the scalings $\mathrm{V}_{\mathrm{sh}} \propto \mathrm{E}_{\mathrm{z}}{ }^{4}$ and $\Delta \propto \mathrm{E}_{\mathrm{z}}{ }^{3}$. In general, the linear rf wave solver will have to iterate, e.g. varying the sheath width, until these nonlinear relationships are satisfied. Since the BC is applied locally at each boundary grid point in the rf wave or antenna code, one would obtain the spatial distribution of the sheath quantities $\left(\Delta, \mathrm{V}_{\mathrm{sh}}\right.$, and $\mathrm{P}_{\mathrm{sh}}$ ) on the boundary as part of the rf field solution.

There are several circumstances in which this iteration procedure is expected to lead to significant sheath power dissipation and modification in the self-consistent rf fields near the boundary: (i) near-field (antenna) sheaths driven by the mismatch between the magnetic field and the antenna orientation; (ii) far-field sheaths due to the mismatch between the wall and neighboring flux surfaces ${ }^{14}$ in cases of poor single pass absorption; and (iii) formation of coaxial modes around the low density region of the tokamak, causing large rf electric fields in the vicinity of the wall. 


\section{Validity Conditions and Relation to Other Models}

To conclude this section, we summarize and discuss the conditions for applicability of the sheath BC and power dissipation analysis in rf codes.

The theory of electron-poor rf sheaths underlying the present work is valid in the frequency regime $\omega_{\mathrm{pi}} \sin \alpha<\omega<\omega_{\mathrm{pe}} \sin \alpha$, which implies immobile ions and inertia-free electrons, and for equilibrium magnetic fields satisfying $\sin \alpha=\mathbf{n} \cdot \mathbf{b}>\left(\mathrm{m}_{\mathrm{e}} / \mathrm{m}_{\mathrm{i}}\right)^{1 / 2}$. The inertia-free electron assumption is closely related to the condition for MaxwellBoltzmann electrons in the sheath, $\omega<\mathrm{v}_{\mathrm{e}} \sin \alpha / \Delta$, which is also required by the theory. The conditions for validity of the sheath theory in various regimes are discussed in more detail in earlier references.6,21,22 The assumption of inertia-free, Maxwell-Boltzmann electrons can be restrictive in the LH frequency range (see Appendix B); our main interest here is in the ion cyclotron range of frequency (ICRF) regime.

The electrostatic approximation in the sheath region is justified in the "thin sheath" limit, $\mathrm{k}_{\mathrm{t}} \Delta<<1$. We also apply the "static sheath" approximation ( $\Delta$ constant in time) here, which neglects sideband (multiple harmonic) and Fermi acceleration effects, because we are only interested in the $\mathrm{BC}$ on the fundamental rf field component. (It should be noted, however, that rf sheaths have a significant second harmonic component as well.) The form of the sheath term $\propto \Delta$ in the sheath BC, Eq. (9), assumes that the rf electrical current is carried across the sheath by the displacement current (as opposed to particle currents), which requires immobile ions, $\omega>\omega_{\mathrm{pi}} \sin \alpha$.

Next, we consider the condition for modeling the rf properties of the sheath response as a resistive vacuum layer with the dielectric given in Eq. (1). The assumption $\operatorname{Re}\left[\varepsilon_{\mathrm{sh}}\right]=1$ is valid if the sheath potential is positive with respect to the wall and set by the requirement of suppressing the otherwise large parallel electron current to the wall (resulting in an electron-poor sheath). This imposes the condition on the field angle discussed previously, viz. that $\sin \alpha>\left(\mathrm{m}_{\mathrm{e}} / \mathrm{m}_{\mathrm{i}}\right)^{1 / 2}$ [See Eq. (15)]. In this limit the sheath response to rf waves is primarily capacitive. Thus, the rf field solutions obtained from the 
resistive dielectric model are expected to be self-consistent when $v<<1$. From Eqs. (13) - (18), we obtain the scaling $v \sim 1 / E_{z}$, which suggests that this approximation works better for larger rf fields. For the model problem discussed in Sec. III, we demonstrate that the sheath BC formulated here gives self-consistent power dissipation

The expressions for the sheath width and sheath power dissipation given in Eqs. (13) and (16) are local, but require the dc sheath potential $V_{0}$ which can in principle be non-local. This comes about because $\mathrm{V}_{0}$ (and hence $\mathrm{C}_{\mathrm{sh}}$ ) are always determined by the global requirement of no net time-averaged current flowing out of the system. Both the computer simulation in Ref. 6 and the analytic derivations $13,21,22$ used parallel plate capacitor models with two symmetric plates (e.g. anti-symmetric voltages and equal areas) to derive $\mathrm{C}_{\mathrm{sh}}$. While in principle these calculations coupled the sheaths at each end of the field line, the plasma screening of the electric field in the plasma between the sheaths (e.g. see Sec. III and Fig. 5) and the symmetry assumption allow the results at each sheath to be interpreted as if the sheaths were uncoupled from each other. More generally, with asymmetric voltages and/or areas at each end of the field line, the resulting dc sheath potentials at both ends can be either coupled or uncoupled, depending on the degree to which the parallel plasma resistivity can support a dc parallel potential gradient along the field line. The dc-coupled (low resistivity) case was considered in Ref.29. In this case $\mathrm{V}_{0}$ is strictly constant along $\mathrm{B}$ and depends on a combination of the rf voltages and areas at each sheath. In the present paper, for simplicity, we restrict the discussion to the uncoupled (large resistivity) case, where each end of the field line can have a different, locally-determined $\mathrm{V}_{0}$.

Finally, we note that the scalings used here for sheath rectification $V_{0} / V_{\text {sh }}$, sheath width $\Delta$, sheath capacitance $\mathrm{C} \propto \varepsilon_{\mathrm{sh}} / \Delta$, and power dissipation $\mathrm{P}_{\text {sh }}$ agree with other theories 21,22 in the high voltage limit $\left(\mathrm{eV}_{\mathrm{sh}} / \mathrm{T}_{\mathrm{e}}>>1\right)$, but the numerical factors are slightly different. Other models, which give a more accurate description of the moderate voltage cases, can be easily incorporated into this BC for different applications. For 
example, work is in progress ${ }^{24}$ to implement a similar sheath BC using the GodyakLieberman sheath models 21,22 for plasma processing. A paper by Jaeger et al. ${ }^{23}$ on modeling power dissipation in high-density inductively coupled plasma sources also incorporated the Lieberman sheath model ${ }^{21}$ but took a different approach to the present one. Instead of applying the vacuum sheath model to derive an "external" BC in the direction normal to the sheaths, it was used in Ref. 23 to derive an effective conductivity for the interior grid cell that contains the wall-sheath boundary. (This is useful for numerical solution techniques which require that the radial computational domain be periodic.) One can derive the effective conductivity by reinterpreting Eq. (10) as applying to wall and sheath regions of widths $\Delta_{1}=(1-\delta) \Delta \mathrm{z}$ and $\Delta_{2}=\delta \Delta \mathrm{z}$, respectively. Adding voltages and using the fact that the normal component of $\mathbf{D}$ is constant across regions (here, $\mathrm{D}_{\mathrm{z}}=\varepsilon_{\mathrm{zz}} \mathrm{E}_{\mathrm{z}}=$ const.), one obtains

$$
\frac{\Delta_{1}+\Delta_{2}}{\varepsilon_{\mathrm{zz}, \mathrm{eff}}}=\frac{\Delta_{1}}{\varepsilon_{\mathrm{zz}, 1}}+\frac{\Delta_{2}}{\varepsilon_{\mathrm{zz}, 2}}
$$

This reformulation of the BC should be physically equivalent to the approach taken here.

\section{EPW Model Problem}

In this section, we illustrate the physics of the sheath BC by solving a model problem analytically in simple geometry. We consider an electron plasma wave (EPW) propagating across the magnetic field $\left(\mathbf{B}_{0}=\mathrm{B}_{0} \mathbf{e}_{\mathrm{z}}\right)$ in a waveguide filled by a constant low-density plasma, assuming $\mathrm{n}<\mathrm{n}_{\mathrm{LH}}$ and thus $\varepsilon_{\perp}>0$, where $\mathrm{n}_{\mathrm{LH}}$ is the lower-hybrid (LH) density for specified wave frequency $\omega$. We assume propagation mainly in the $\mathrm{x}$ direction ( $\mathbf{k}=\mathrm{k}_{\mathrm{x}} \mathbf{e}_{\mathrm{x}}+\mathrm{k}_{\mathrm{z}} \mathbf{e}_{\mathrm{z}}, \mathrm{k}_{\mathrm{x}}>>\mathrm{k}_{\mathrm{z}}$ ), which is the axis of the waveguide. For the geometry assumed here (see Fig. 2) $\mathbf{B}_{0}$ is perpendicular to the waveguide walls so that $-\mathbf{n}$ $= \pm \mathbf{b}$ at $\mathrm{z}= \pm \mathrm{L}$. In this simple geometry, the wavevectors are given by $\mathrm{k}_{\mathrm{t}}=\mathrm{k}_{\perp}=\mathrm{k}_{\mathrm{x}}$, $\left|\mathrm{k}_{\mathrm{n}}\right|=\mathrm{k}_{\|}=\mathrm{k}_{\mathrm{z}}$, and the area factors in Sec. IIB by $A_{t}=A_{\perp}=A$. The generalization to $\mathrm{a}$ tilted magnetic field is straightforward but unnecessary to illustrate the physics. As the 
mode propagates down the waveguide, it must satisfy the dissipative sheath $\mathrm{BC}$ at the walls of the waveguide and lose energy to the sheaths. This EPW problem is conceptually related to the wave physics in front of an IBW antenna in the low-density region where $\mathrm{n}$ $<\mathrm{n}_{\mathrm{LH}}$. Thus, this problem is relevant to understanding the power coupling issues in experiments using the EPW-IBW mode transformation approach. This treatment may also be relevant to $\mathrm{LH}$ waveguide launchers in tokamaks, although the sheath power dissipation computed here would be smaller at the higher LH frequency (see Appendix B).

\section{A. Linear EPW solution without sheath power dissipation}

Plasma region. We use the ES approximation $(\mathbf{E}=-\nabla \Phi=-\mathrm{ik} \Phi)$ to describe the

electron plasma wave (EPW) solution in the plasma region, valid when $\mathrm{n}_{\|}{ }^{2}>>\varepsilon_{\perp} \sim 1$. The wave satisfies $\nabla \cdot \mathbf{D}=0$, and the desired analytic solution $\Phi(\mathrm{z})$ is even about the midplane of the waveguide ( $\mathrm{z}=0$ ), so that only the solution for $\mathrm{z} \geq 0$ need be given. In the plasma $(-\mathrm{L}<\mathrm{z}<\mathrm{L})$, we use the ansatz

$$
\Phi^{(\mathrm{pl})}(\mathrm{z})=\Phi_{0}(\mathrm{x}) \cos \mathrm{k}_{\mathrm{z}} \mathrm{z} \mathrm{e}^{\mathrm{i} \theta},
$$

with $\Phi_{0}(\mathrm{x})$ real, $\mathrm{k}_{\mathrm{y}}=0$ and $\theta=\mathrm{k}_{\mathrm{x}} \mathrm{x}-\omega \mathrm{t}$.

The wavenumbers satisfy the ES dispersion relation

$$
\begin{gathered}
\mathrm{k}_{\perp}{ }^{2} \varepsilon_{\perp}+\mathrm{k}_{\|}{ }^{2} \varepsilon_{\|}=0, \\
\varepsilon_{\perp}=1-\frac{\omega_{\mathrm{pi}}^{2}}{\omega^{2}-\Omega_{\mathrm{i}}^{2}}, \varepsilon_{\|}=1-\frac{\omega_{\mathrm{pe}}^{2}}{\omega^{2}} \approx-\frac{\omega_{\mathrm{pe}}^{2}}{\omega^{2}} .
\end{gathered}
$$

Rewriting Eq. (21) letting $\mathrm{k}_{\perp} \rightarrow \mathrm{k}_{\mathrm{x}}$ and $\mathrm{k}_{\|} \rightarrow \mathrm{k}_{\mathrm{z}}$ and assuming low density ( $\varepsilon_{\perp}>0$ ), we obtain

$$
\mathrm{k}_{\mathrm{x}}{ }^{2}=\frac{\mathrm{k}_{\mathrm{z}}{ }^{2} \omega_{\mathrm{pe}}^{2}}{\varepsilon_{\perp} \omega^{2}}>0,
$$


This dispersion relation implies that the EPW is a negative-energy wave that propagates backwards (phase and group velocities in opposite directions), i.e.

$$
\frac{\mathrm{d} \omega}{\mathrm{dk}_{\mathrm{x}}}=-\frac{\omega}{\mathrm{k}_{\mathrm{x}}}
$$

Thus, we choose $k_{x}<0$ to give energy flow in the positive $x$ direction. This root of Eq. (21) is given by

$$
\mathrm{k}_{\mathrm{x}} \mathrm{L}=-\eta\left(\frac{-\varepsilon_{\|}}{\varepsilon_{\perp}}\right)^{1 / 2},
$$

where $\eta \equiv \mathrm{k}_{\mathrm{z}} \mathrm{L}$ and $\varepsilon_{\|}=\varepsilon_{\mathrm{zz}}$.

Application of the sheath boundary condition. The rf field components at the entrance to the sheath $(\mathrm{z}=\mathrm{L})$ is obtained from the solution (20) for $\Phi(\mathrm{pl})$

$$
\begin{aligned}
& \mathrm{E}_{\mathrm{x}}^{(\mathrm{pl})}(\mathrm{L})=-\mathrm{ik}_{\mathrm{x}} \Phi^{(\mathrm{pl})}(\mathrm{L}), \\
& \mathrm{E}_{\mathrm{z}}^{(\mathrm{pl})}(\mathrm{L})=\mathrm{k}_{\mathrm{z}} \sin \left(\mathrm{k}_{\mathrm{z}} \mathrm{L}\right) \Phi^{(\mathrm{pl})}(\mathrm{L}),
\end{aligned}
$$

where we define $\Phi^{(\mathrm{pl})}(\mathrm{L}) \equiv \Phi_{\mathrm{L}}$ and note that $\mathrm{E}_{\mathrm{z}}^{(\mathrm{pl})}=\mathrm{E}_{\mathrm{n}}^{(\mathrm{pl})}$ and $\mathrm{E}_{\mathrm{x}}^{(\mathrm{pl})}=\mathrm{E}_{\mathrm{t}}^{(\mathrm{pl})}$. At this point we employ the sheath BC, avoiding the need for an explicit solution for $\Phi$ in the sheath region. Substituting the fields into Eq. (9) gives an eigenmode localization condition which determines $\eta$ as a function of $\Lambda$

$$
-\Lambda \eta \tan \eta=1
$$

where

$$
\Lambda \equiv-\frac{\varepsilon_{\mathrm{nn}}^{(\mathrm{pl})}}{\varepsilon_{\mathrm{nn}}^{(\mathrm{sh})}} \frac{\Delta}{\mathrm{L}} \approx \frac{\omega_{\mathrm{pe}}^{2}}{\omega^{2}} \frac{\Delta}{\mathrm{L}},
$$

$\varepsilon_{\mathrm{nn}}^{(\mathrm{sh})}=1$ in the vacuum sheath model (neglecting dissipation, $v=0$ ), and $\varepsilon_{\mathrm{nn}}^{(\mathrm{pl})}=\varepsilon_{\|}^{(\mathrm{pl})} \approx-\omega_{\mathrm{pe}}{ }^{2} / \omega^{2}$. The constraint (27) expresses the continuity of $\Phi$ and $\mathrm{D}_{\mathrm{z}}$ across the sheath-plasma boundary for the EPW solution. We define $\Lambda$ to be real and 
positive for the regime in which the EPW propagates $\left(\varepsilon_{\perp}^{(\mathrm{pl})}>0\right.$, $\left.\varepsilon_{\|}^{(\mathrm{pl})}<0\right)$, which requires sufficiently low density, viz. $\omega>\omega_{\mathrm{LH}}$.

A useful identity that follows from Eq. (27) is

$$
\frac{\Lambda \eta^{2}}{\cos ^{2} \eta}=-\frac{2 \eta}{\sin 2 \eta}
$$

It can be seen from Eq. (29) that the solution of Eq. (27) for the lowest waveguide eigenmode gives $\eta$ in the range

$$
\frac{\pi}{2}<\eta<\pi
$$

resulting in $\Phi_{\mathrm{L}}<0$ [see Eq. (20)], as shown in Fig. 3. Physically, this occurs because $\varepsilon_{\mathrm{zz}}$ changes sign across the (vacuum) sheath-plasma boundary, implying that $E_{z}=-i$ $\mathrm{k}_{\mathrm{z}} \Phi$ must also change sign to maintain constant $\mathrm{D}_{\mathrm{z}}$ across the boundary. Analytical expressions for $\eta$ have been obtained in the following limiting cases

$$
\begin{array}{ll}
\eta=\frac{\pi}{2}(1+\Lambda), & (\Lambda<<1) \\
\eta=\pi-\frac{1}{\pi \Lambda} \quad . & (\Lambda>>1)
\end{array}
$$

The BC at a conducting surface, $\mathbf{E}_{\mathrm{t}}=-\mathrm{i} \mathrm{k}_{\mathrm{t}} \Phi=0$, corresponds to $\eta \equiv \mathrm{k}_{\mathrm{z}} \mathrm{L}=\pi / 2$, consistent with taking the limit $\Lambda \rightarrow 0$ in Eq. (31).

It is useful to illustrate this solution with a sketch of the eigenmode [see Fig. 3], adding the sheath region to show the whole picture. The linear dependence of $\Phi(\mathrm{z})$ in the sheath region is built into the SBC and does not need to be explicitly solved for. In numerical solutions, this is a great benefit because resolving the sheath requires a large number of grid points and is generally not practical.

We also mention in passing that another class of modes ("sheath-plasma waves") arises from the sheath $\mathrm{BC}$. These modes are localized to the sheath-plasma interface ${ }^{30}$ 
and are obtained in the limit of pure imaginary $\eta$ satisfying $|\eta|>>1$. The sheath plasma waves are not coupled to the EPW, and play no role in the present calculation.

The sheath voltage $\mathrm{V}_{\text {sh }}$ was defined in Eq. (17) and its magnitude is related to $\eta$ by

$$
\mathrm{V}_{\mathrm{sh}}=\left|\Phi_{\mathrm{L}}\right|=\Phi_{0}|\cos \eta|, \quad \eta \equiv \mathrm{k}_{\mathrm{z}} \mathrm{L}
$$

It can be seen from Eqs. (26), (31) and (32) that $\Lambda \sim 1$ is a typical condition for sheath effects to matter. When $\Lambda \rightarrow 0$ (implying $\eta \rightarrow \pi / 2$ ), the rf fields satisfy the metal wall $\mathrm{BC}$ and the sheath potential vanishes $\left(\mathrm{V}_{\mathrm{sh}} \rightarrow 0\right)$. When $\Lambda \rightarrow \infty(\eta \rightarrow \pi), \Phi(\mathrm{z})$ is constant in the plasma; in this limit, the $\mathrm{E}_{\mathrm{z}}=\mathrm{E}_{\|}$component is completely screened from the plasma and appears across the sheath. Also in this limit the sheath potential obtains its maximum value $\left(\mathrm{V}_{\mathrm{sh}}=\Phi_{0}\right)$.

\section{B. Nonlinear equation for wave decay and sheath power dissipation}

The generalization of Eq. (27) to include weak dissipation is

$$
-\Lambda \eta \tan \eta=1+\mathrm{i} v
$$

This constraint can be solved perturbatively with $\eta=\eta_{0}+\eta_{1}$, where $\eta_{0}=\operatorname{Re}\left[k_{z}\right] L$ is the solution to Eq. (27) and $\eta_{1}=\operatorname{Im}\left[\mathrm{k}_{\mathrm{z}}\right] \mathrm{L}<<\eta_{0}$. The solution can be put in the form

$$
\frac{\eta_{1}}{\eta_{0}}=\frac{i v \sin 2 \eta_{0}}{\sin 2 \eta_{0}+2 \eta_{0}}
$$

which implies that $\eta_{1}=\operatorname{Im} \eta$ is negative because $-1<\sin 2 \eta_{0} / 2 \eta_{0}<0$. At this point, we drop the subscript 0 on $\eta_{0}$. In the small-v limit, it follows from Eq. (25) that

$$
\frac{\operatorname{Im}_{\mathrm{x}}}{{\operatorname{Re~} \mathrm{k}_{\mathrm{x}}}}=\frac{\operatorname{Imk_{\mathrm {z}}}}{\operatorname{Re} \mathrm{k}_{\mathrm{z}}}=\frac{\operatorname{Im} \eta}{\operatorname{Re} \eta}
$$

Thus, Eq. (35) with $\operatorname{Im} \eta<0$, Re $\eta>0$ and $\operatorname{Re} k_{x}<0$ implies that $\operatorname{Im} k_{x}>0$ so that the wave decays as it propagates. 
The rate of decay is proportional to $v$ and is determined by the absorption mechanism: in this case, power dissipation due to ion acceleration in the sheaths [Eq. (16)]. Since this problem is nonlinear, we will employ a generalization of the WKB method using a two-scale expansion to obtain an equation for the amplitude $\Phi_{0}(\mathrm{x})$. All equilibrium quantities are assumed to be spatially constant in this calculation.

In the plasma region $\left(\Phi^{(\mathrm{pl})} \rightarrow \Phi\right)$, the eigenmode equation has the form [see Eq. (21)]

$$
\varepsilon_{\perp} \nabla_{\mathrm{x}}^{2} \Phi=-\varepsilon_{\|} \nabla_{\mathrm{z}}^{2} \Phi
$$

and we make the two-space-scale ansatz

$$
\Phi=\phi(x, z) e^{\mathrm{i} \theta},
$$

where $\phi(\mathrm{x}, \mathrm{z})=\Phi_{0}(\mathrm{x}) \cos \left(\mathrm{k}_{\mathrm{z}} \mathrm{z}\right)$ and $\theta=\operatorname{Re}\left(\mathrm{k}_{\mathrm{x}}\right) \mathrm{x}-\omega \mathrm{t}$. Here, $\phi(\mathrm{x}, \mathrm{z})$ is a real function, separable in $\mathrm{x}$ and $\mathrm{z}$, and a slow function of $\mathrm{x}$, i.e. $\nabla_{\mathrm{x}} \ln \phi(\mathrm{x}, \mathrm{z})<<\mathrm{k}_{\mathrm{x}}$. We will treat $\nabla_{\mathrm{x}} \ln \phi(\mathrm{x}, \mathrm{z}), \nabla_{\mathrm{x}} \mathrm{k}_{\mathrm{x}}$ and $\nabla_{\mathrm{x}} \eta$ as first order in $\mathrm{v}$, so that the dissipation drives the slow decay of the wave in $x$.

In lowest order, the eigenmode equation reduces to the dispersion relation (21); in next order, we obtain

$$
\begin{gathered}
2 \mathrm{ik}_{\mathrm{x}} \nabla_{\mathrm{x}} \phi+\mathrm{i} \phi \nabla_{\mathrm{x}} \mathrm{k}_{\mathrm{x}}=\frac{\varepsilon_{\|}}{\varepsilon_{\perp}} \frac{2 \eta \eta_{1}}{\mathrm{~L}^{2}} \phi, \\
\Rightarrow \quad \nabla_{\mathrm{x}}\left(\mathrm{k}_{\mathrm{x}} \phi^{2}\right)=\frac{\eta_{1}}{\eta} 2 \mathrm{ik}_{\mathrm{x}}^{2} \phi^{2},
\end{gathered}
$$

where the second form was obtained by multiplying by $\phi$ and using Eq. (25) to simplify the last term. Next, we integrate over the interval $\mathrm{z}=(-\mathrm{L}, \mathrm{L})$ to obtain an equation for the x-dependent part of $\Phi$ using the identity

$$
\int_{-L}^{\mathrm{L}} \mathrm{dz} \phi^{2}=\Phi_{0}^{2} \mathrm{~L}\left(\frac{\sin 2 \eta+2 \eta}{2 \eta}\right) .
$$


Note that $\eta$ is a function of $x$ through Eq. (27), because the sheath width $\Delta$ (and hence $\Lambda$ ) decreases as the mode decays. Thus, combining Eqs. (38) and (39), we obtain

$$
\nabla_{x}\left(\frac{\sin 2 \eta+2 \eta}{2 \eta} \mathrm{k}_{\mathrm{x}} \Phi_{0}^{2}\right)=\frac{2 \mathrm{i} \eta_{1}}{\eta} \frac{\sin 2 \eta+2 \eta}{2 \eta} \mathrm{k}_{\mathrm{x}}^{2} \Phi_{0}^{2}
$$

Finally, using Eq. (34) for $\eta_{1}$ and the fact that $k_{x} / k_{z}$ is independent of $x$ by Eq. (23), after some algebra we obtain

$$
\frac{\mathrm{d}}{\mathrm{dx}}\left((\sin 2 \eta+2 \eta) \Phi_{0}^{2}\right)=-\frac{2 v}{\mathrm{~L}} \frac{\mathrm{k}_{\mathrm{x}}}{\mathrm{k}_{\mathrm{z}}} \eta \sin 2 \eta \Phi_{0}^{2}
$$

This is the governing equation that determines the decay of $\Phi_{0}(\mathrm{x})$ due to the sheath power losses, which are proportional to $v$. Analytic and numerical solutions of Eq. (41) will be discussed in the next section, where $v$ will be expressed in terms of $\Phi_{0}$ using the specific scaling $\mathrm{P}_{\mathrm{sh}} \propto \Phi_{0}$ [see Eq. (16)]. It is also important to check that Eq. (41) satisfies energy conservation. In Appendix A, we demonstrate that Eq. (41) satisfies the Poynting Theorem for steady-state energy conservation in the limit $v<<1$ but for arbitrary $\eta \equiv \mathrm{k}_{\mathrm{z}} \mathrm{L}$.

\section{Solution of the wave equation}

Rescaled equation. We scale $\mathrm{x}$ to $\mathrm{L}_{\mathrm{X}}=-\mathrm{Lk}_{\mathrm{Z}} /\left(\mathrm{k}_{\mathrm{X}} \mathrm{v}_{0}\right)$ (noting that $\mathrm{k}_{\mathrm{X}}<0$ ) and drop the subscript 0 on $\Phi$. We see that $\Lambda \propto \Delta \propto\left(\mathrm{V}_{\mathrm{sh}}\right)^{3 / 4}$ for a high voltage sheath by Eqs. (13) and (28). Also, the power dissipation $\mathrm{P} \propto v\left(\mathrm{~V}_{\mathrm{sh}} / \Delta\right)^{2} \Delta$ is due to the energy loss by ions falling down the sheath (with energy $\propto \mathrm{V}_{\mathrm{sh}}$ ), so that $v \propto \Delta / \mathrm{V}_{\mathrm{sh}} \sim 1 /\left(\mathrm{V}_{\mathrm{sh}}\right)^{1 / 4}$, where $\mathrm{V}_{\text {sh }}=\Phi|\cos \eta|$ by Eq. (32). To make these nonlinear dependences explicit, we introduce the following normalizations

$$
\Lambda \equiv\left|\frac{\Phi \cos \eta}{\Phi_{\Lambda}}\right|^{3 / 4}, \quad v \equiv v_{0}\left|\frac{\Phi_{\Lambda}}{\Phi \cos \eta}\right|^{1 / 4}
$$


which implicitly defines $\Phi_{\Lambda}$ and $v_{0}$ in terms of constants. Combining these definitions with the expressions for $v$ and $\Lambda$ in Eqs. (18) and (28), we find that the coefficients are given by

$$
\frac{\mathrm{e} \Phi_{\Lambda}}{\mathrm{T}_{\mathrm{e}}}=\left(\frac{\mathrm{L}}{\left|\varepsilon_{\|}\right| \lambda_{\mathrm{D}}}\right)^{4 / 3} \quad, \quad v_{0}=2 \mathrm{C}_{\mathrm{sh}}\left(\frac{\mathrm{m}_{\mathrm{e}}}{\mathrm{m}_{\mathrm{i}}}\right)^{1 / 2}\left(\frac{\lambda_{\mathrm{D}}{ }^{1 / 3}\left|\varepsilon_{\|}\right|^{5 / 6}}{\mathrm{~L}^{1 / 3}}\right)
$$

where $C_{s h}$ is the sheath rectification coefficient defined in Eq. (14). Normalizing $\Phi$ to $\Phi_{\Lambda}$, we have that $\Lambda=|\Phi \cos \eta|^{3 / 4}, \nu=v_{0} /\left.\Phi \cos \eta\right|^{1 / 4}$. Substituting all of these scalings into Eq. (41), we obtain

$$
\frac{\mathrm{d}}{\mathrm{dx}}\left((\sin 2 \eta+2 \eta) \Phi^{2}\right)=\frac{2 \eta \sin 2 \eta}{|\cos \eta|^{1 / 4}} \Phi^{7 / 4}
$$

which, we reiterate, is valid in the high voltage sheath limit, $\mathrm{eV}_{\mathrm{sh}}>\mathrm{T}$.

We see that the problem has only one parameter, the initial amplitude of $\Phi$ at $\mathrm{x}=$ 0 which we denote here as $\Phi_{\max }=\Phi(\mathrm{x}=0)$. Since the solution is decaying, it is useful to solve for the case $\Phi_{\max }>>1$ and cases with smaller amplitudes can be inferred from just considering the starting point to be at $x>0$. Thus, the problem is reduced to that of finding a single universal solution.

Asymptotic solutions. Since $\Phi(\mathrm{x})$ is decaying, we consider the asymptotic limit $\Phi \rightarrow 0$ as $\mathrm{x} \rightarrow \infty$. The scaling in Eq. (42) implies $\Lambda<<1$, and Eq. (31) gives $\eta \approx(\pi / 2)(1+\Lambda)$ so that $\sin 2 \eta \approx-\pi \Lambda, \cos \eta \approx-\pi \Lambda / 2$, and $\Lambda=(\pi \Phi / 2)^{3}$ so that Eq. (44) reduces to

$$
\frac{\mathrm{d} \Phi}{\mathrm{dx}}=-\left(\frac{\pi \Phi}{2}\right)^{3}
$$

The solution of this equation is

$$
\Phi \sim \frac{2}{\pi^{3 / 2} \mathrm{x}^{1 / 2}}
$$


which exhibits the expected decay as $\mathrm{x} \rightarrow \infty$.

The other asymptotic limit $(\Phi \rightarrow \infty)$ can be relevant near the waveguide entrance. In this case, using $\Lambda>>1$ and Eq. (31), we have that $\eta \approx \pi-1 / \pi \Lambda$ and $\sin 2 \eta \approx-2 / \pi \Lambda$, $\cos \eta \approx-1$, and $\Lambda=\Phi^{3 / 4}$ so that Eq. (44) reduces to

$$
\frac{\partial}{\partial \mathrm{x}} \Phi=-\frac{1}{\pi}
$$

which gives linear decay in $\mathrm{x}$. It follows from the asymptotic results that the location $\mathrm{x}_{\mathrm{t}}$ of the transition point where the solution $\Phi(\mathrm{x})$ transitions from Eq. (47) to Eq. (46) is given approximately by $\mathrm{x}_{\mathrm{t}}=\pi \Phi_{\max }-2 /\left(\pi \Phi_{\max }^{1 / 2}\right)$.

Numerical solution. To summarize the problem, the equations to be solved are

$$
\begin{gathered}
\frac{\mathrm{d}}{\mathrm{dx}}\left((\sin 2 \eta+2 \eta) \Phi^{2}\right)=\frac{2 \eta \sin 2 \eta}{|\cos \eta|^{1 / 4}} \Phi^{7 / 4} \\
-\Lambda \eta \tan \eta=1 \\
\Lambda=|\Phi \cos \eta|^{3 / 4}
\end{gathered}
$$

subject to the initial condition $\Phi(\mathrm{x}=0)=\Phi_{\max }$. Here, $\Phi$ is normalized to $\Phi_{\Lambda}$ and $\mathrm{x}$ is normalized $\mathrm{L}_{\mathrm{X}}=-\mathrm{L} \mathrm{k}_{\mathrm{Z}} / \mathrm{k}_{\mathrm{X}} v_{0}$.

The numerical solution for $\Phi(\mathrm{x})$ is illustrated by the solid curve in Fig. 4 for the case $\Phi_{\max }=2$, which shows that the wave decays as a result of the sheath power dissipation. The numerical solution recovers both of the asymptotic limits and shows that, in fact, the strong-sheath (linear decay) regime holds over a wide range of $\Phi$. Also shown in Fig. 4, the curve with long dashes depicts the rectified (dc) potential $\mathrm{V}_{0}(\mathrm{x}) \equiv \mathrm{C}_{\mathrm{sh}} \mathrm{V}_{\mathrm{sh}}=\mathrm{C}_{\mathrm{sh}} \Phi|\cos \eta|$ (neglecting the Bohm sheath contribution) and the curve with short dashes shows the rate at which the initial energy in the Poynting flux is lost to the sheaths. Specifically, we plot the ratio $\mathrm{R}_{\mathrm{P}}$ of the total sheath power dissipation $\mathrm{P}_{\mathrm{sh}}$ 
(integrated over the wave trajectory in $\mathrm{x}$ ) to the Poynting flux $\mathrm{S}_{\mathrm{x}}$ at $\mathrm{x}=0$ (summed over both the plasma and the two sheaths):

$$
R_{P}=\frac{\int_{0}^{x} d x^{\prime} P_{s h}\left(x^{\prime}\right)}{\int_{-L}^{L} d z S_{x}(x=0)}
$$

The quantities $\mathrm{P}_{\mathrm{sh}}$ and $\mathrm{S}_{\mathrm{x}}$ are defined and calculated in Appendix A [see Eqs. (A9) and (A7)].

Figure 4 illustrates how all of the initial energy in the wave is lost to the sheaths if the wave propagates far enough. Thus, $\mathrm{R}_{\mathrm{P}} \rightarrow 1$ as $\mathrm{V}_{0} \rightarrow 0$, consistent with the fact that the calculated sheath power loss mechanism is due to ions accelerated in the potential $\mathrm{eV}_{0}$. The distance required for this to happen depends on the initial amplitude $\left(\Phi_{\max }\right)$ in this nonlinear problem. For the case shown here $\left(\Phi_{\max }=2\right)$ the EPW loses about $30 \%$ of its initial energy in the distance $L_{\mathrm{X}}=-\mathrm{Lk}_{\mathrm{Z}} / \mathrm{k}_{\mathrm{X}} \mathrm{v}_{0}$. Dimensional estimates of the distance for significant power loss are given in Sec. III D.

The sheath BC also modifies the rf electric field solution in the plasma near the sheath at $\mathrm{z}=\mathrm{L}$. This is illustrated by computing the ratio of the field $\mathrm{E}_{\mathrm{z}}^{(\mathrm{pl})}$ obtained using the sheath $\mathrm{BC}$ to the field $\mathrm{E}_{\mathrm{z} 0}^{(\mathrm{pl})}$ obtained using the metal wall $\mathrm{BC}(\Lambda=0)$. The ratio $\mathrm{E}_{\mathrm{z}}^{(\mathrm{pl})} / \mathrm{E}_{\mathrm{z} 0}^{(\mathrm{pl})} \equiv \sin \eta / \sin (\pi / 2)=\sin \eta \quad$ [see Eq. (26)] is plotted vs $\Phi$ in Fig. 5 with $\mathrm{E}_{\mathrm{z}}$ evaluated near the sheath $(\mathrm{z}=\mathrm{L})$. Figure 5 shows that the rf electric field in the plasma weakens as $\Phi$ increases because of the influence of the sheath BC; conversely, the rf field in the sheath grows because $\mathrm{E}_{\mathrm{Z}}^{(\mathrm{sh})} \propto \Phi / \Delta \sim \mid \Phi \cos \eta^{1 / 4}$. Thus, the sheaths "short out" or screen $\mathrm{E}_{\mathrm{Z}}$ in the plasma.

The scaling $\Lambda \propto \Delta \propto|\Phi \cos \eta|^{3 / 4}$ shows that the sheath BC effect (measured by $\Lambda$ ) increases as the wave amplitude $\Phi$ grows. Using the analytic result $\cos \eta \approx$ $-(\pi / 2)^{4} \Phi^{3}$ obtained in the $\Phi \rightarrow 0$ limit [see the discussion before Eq. (46)], we can estimate the threshold value of $\Phi$ at which $\mathrm{E}_{\mathrm{z}}^{(\mathrm{pl})} / \mathrm{E}_{\mathrm{z} 0}^{(\mathrm{pl})}$ begins to decrease significantly in 
Fig. 5. A $10 \%$ decrease in $\mathrm{E}_{\mathrm{z}}^{(\mathrm{pl})} / \mathrm{E}_{\mathrm{z} 0}^{(\mathrm{pl})}$ requires $\sin \eta=0.9$, implying $\cos \eta=0.44 \approx$ $-(\pi / 2)^{4} \Phi^{3}$ and hence $\Phi \approx 0.4$, in agreement with Fig. 5. Thus, sheath effects become important when the dimensionless $\Phi$ is order unity (dimensional $\Phi \sim \Phi_{\Lambda}$ ).

To summarize this discussion, Figs. 4 and 5 illustrate the main effects of the sheath BC. Both the power lost by the EPW as it propagates [Fig. 4] and the modification of the wave fields near the boundary [Fig. 5] are consequences of imposing the sheath BC in Eq. (9) in the solution for the self-consistent rf field amplitudes.

The curves in Figs. 4 and 5 are universal in the sense that they depend on only one parameter, $\Phi(\mathrm{x}=0)=\Phi_{\max }$. The dependence on the other physical parameters (necessary to convert to dimensional units) is contained in the quantities $L_{X}, \Phi_{\Lambda}$ and $v_{0}$. For a particular physical case, one must calculate these quantities and check whether the validity conditions for the theory are met. For example, the condition for the validity of our perturbative (two-length scale) solution is that $\operatorname{Im} k_{X} / \operatorname{Re}_{\mathrm{X}}<<1$. The general validity condition for the treatment of the sheath power dissipation in the sheath $\mathrm{BC}$ is that $v<<1$. These conditions and the translation of the solution to dimensional parameters are discussed in Sec. III D.

\section{Validity Conditions and Dimensional Quantities}

For the first validity condition, the ratio $\operatorname{Im~} \mathrm{k}_{\mathrm{X}} / \mathrm{Re} \mathrm{k}_{\mathrm{x}}$ can be evaluated using Eqs. (34) and (35) to obtain $\operatorname{Im} k_{x} / \operatorname{Re} k_{x}=\operatorname{Im} \eta / \operatorname{Re} \eta=C_{\eta} v_{0} / \Phi^{1 / 4}$, where $C_{\eta}=$ (cos $\eta)^{-1 / 4} \sin 2 \eta /(\sin 2 \eta+2 \eta)$. In the limit $\Phi>>1$, one finds that $\eta \rightarrow \pi$ and $C_{\eta} \rightarrow 0$, so the two-scale approximation is well satisfied in this limit. As $\Phi \rightarrow 1$, a numerical solution shows that $C_{\eta} \rightarrow 0.15$, which is sufficient for present purposes. (Here, $\Phi$ is the normalized potential shown in Fig. 4; for brevity we do not unfold the scaling with $\Phi_{\Lambda}$.) For a D plasma with $\mathrm{B}=30 \mathrm{kG}, \mathrm{T}_{\mathrm{e}}=20 \mathrm{eV}$ and $\mathrm{L}=50 \mathrm{~cm}$, we have the scaling $\mathrm{v}_{0}=0.25$ $\left(\mathrm{n}_{\mathrm{e}} / 10^{10} \mathrm{~cm}^{-3}\right)^{2 / 3}$. Combining these results and using $\mathrm{n}_{\mathrm{e}}=5 \times 10^{10} \mathrm{~cm}^{-3}$, one obtains the estimate $\operatorname{Im} \eta / \operatorname{Re} \eta \approx 0.1 / \Phi^{1 / 4}$, implying that the two-scale approximation is valid 
at this density for $\Phi>1$, i.e. for normalized $x<3$ in Fig. 4. Thus, at this density the solution is valid in most of the region in which the sheath power losses occur.

The second condition (on the sheath power dissipation) is more restrictive. For $\Phi \geq 1$, we can make the approximation $|\cos \eta| \approx 1$ so that $v=v_{0} / \Phi^{1 / 4}$ by Eq. (42). For the base case parameters given above, $v_{0}<1$ for $n_{e}<8 \times 10^{10} \mathrm{~cm}^{-3}$ and $v<<1$ is only marginally satisfied for $\Phi>1$ at this density. Nonetheless, the model is useful for estimating when an order unity fraction of the incident power can be dissipated in the sheaths.

Finally, we return to the discussion of power loss and derive a dimensional form for the decay length. If we define significant power loss to be a factor 2 reduction in the initial amplitude $\Phi_{\text {max }}$, then the propagation distance $\mathrm{L}_{\text {loss }}$ to incur this loss is

$$
\mathrm{L}_{\text {loss }}=\frac{\pi}{2} \Phi_{\max } \mathrm{L}_{\mathrm{x}}=\frac{\pi \lambda_{\mathrm{D}}}{4 \mathrm{C}_{\mathrm{sh}}}\left(\frac{\mathrm{m}_{\mathrm{i}}}{\mathrm{m}_{\mathrm{e}}}\right)^{1 / 2}\left(\frac{\mathrm{e} \Phi}{\mathrm{T}}\right)
$$

where the last form of Eq. (50) uses the definition $L_{X}=-L_{z} /\left(k_{x} v_{0}\right)$, Eqs. (43) for $v_{0}$ and $\mathrm{e} \Phi_{\Lambda} / \mathrm{T}_{\mathrm{e}}$, and assumes $\varepsilon_{\perp} \sim 1$. In a slight departure from the notation of the rest of this section, the quantities $\mathrm{L}_{\text {loss }}, \mathrm{L}_{\mathrm{X}}$ and $\Phi$ are dimensional in Eq. (50). For ICRF frequencies with $\mathrm{C}_{\mathrm{sh}} \sim 1$, an excellent approximation to Eq. (50) is just

$$
\mathrm{L}_{\text {loss }} \sim\left(\frac{\mathrm{m}_{\mathrm{i}}}{\mathrm{m}_{\mathrm{e}}}\right)^{1 / 2} \Delta
$$

since $\pi /\left(4 \mathrm{C}_{\mathrm{sh}}\right) \sim 1, \Delta=\lambda_{\mathrm{D}}(\mathrm{e} \Phi / \mathrm{T})^{3 / 4}$ and for reasonable parameters $(\mathrm{e} \Phi / \mathrm{T})^{1 / 4} \sim 1$. For example, taking $\mathrm{T}_{\mathrm{e}}=20 \mathrm{eV}$ and $\mathrm{n}_{\mathrm{e}}=10^{11} \mathrm{~cm}^{-3}$ gives a Debye length $\lambda_{\mathrm{D}}=0.01 \mathrm{~cm}, \mathrm{a}$ sheath width $\Delta=0.03 \mathrm{~cm}$ for $\mathrm{e} \Phi / \mathrm{T} \sim 4$ (e.g. consider an $80 \mathrm{~V}$ sheath potential in a $20 \mathrm{eV}$ plasma), and a $50 \%$ power loss distance $\mathrm{L}_{\text {loss }} \approx 2 \mathrm{~cm}$. Decreasing the plasma density to $\mathrm{n}_{\mathrm{e}}=5 \times 10^{10} \mathrm{~cm}^{-3}$ with the other parameters held fixed, increases the sheath width and $50 \%$ power loss distance to $\Delta=0.04 \mathrm{~cm}$ and $\mathrm{L}_{\text {loss }}=5 \mathrm{~cm}$. (The dependence of $\mathrm{L}_{\text {loss }}$ on $\mathrm{rf}$ 
frequency is discussed in Appendix B.) Taking for the moment a more general point of view than the model problem, we note that these input parameters were chosen to approximate those in the SOL of tokamaks for IBW heating, and the value of $\mathrm{L}_{\text {loss }}$ obtained from Eq. (51) is comparable to the SOL width. Thus, these estimates suggest that slow waves propagating across the SOL can lose a substantial fraction of the input power to the sheaths, and this can account for the significant loss of heating efficiency in scenarios where a significant SW component is present in the rf fields.

\section{Summary and Discussion}

This paper has presented two main results. In Sec. II, we described an rf sheath boundary condition (SBC) that can be incorporated into rf wave propagation and antenna codes to obtain a self-consistent treatment of the rf sheath physics. The BC is suitable for describing the effect of the sheaths on the linear rf waves [Eq. (9)] and (after iteration or other nonlinear solution methods) the nonlinear sheath power dissipation [Eq.(16)]. The SBC is intended mainly for use in the ICRF frequency regime, but may also be applicable in the LH frequency regime if certain conditions are met (see Appendix B). Although not discussed here, it can be shown that the rf sheath BC is analogous to the low-frequency sheath BC used in calculations of MHD instabilities (e.g. see the brief review in Ref. 31); the rf frequency form discussed here is valid when the particle currents are negligible in the sheaths, as discussed in Sec. IIA. Numerical implementation of the sheath BC was briefly discussed in Sec. IIC.

In Sec. III we presented a calculation of electron plasma wave propagation through a low-density plasma-filled waveguide to check the formalism and illustrate its application. The EPW calculation demonstrates the importance of the sheath BC when the magnetic field has a component normal to the metallic boundary: (i) the SBC modifies the spatial distribution of the propagating wave field, particularly near the sheaths; and (ii) power dissipation due to ions accelerated in the sheaths causes the wave 
amplitude and Poynting flux to decay as it propagates. In rf-heated tokamaks and other fusion devices, the latter effect will decrease the overall heating efficiency and cause heating of material boundaries when sheath voltages are large. In Appendix A, it is shown by explicit calculation for the model EPW problem that energy is conserved in the coupled sheath-plasma system.

The electrostatic EPW model problem illustrates some features of the SBC which are expected to have implications for FW or SW launch using electromagnetic antennas. In past analyses of sheath effects driven by antennas in fusion experiments, ${ }^{3-15}$ the sheath potential was estimated as $\int d s E_{\|}^{\text {vac }}$, where the integral is taken along a field line between the two contact points with the metallic boundary using the "vacuum" rf field component parallel to $\mathbf{B}$. (In the vacuum limit, the rf field distribution along $\mathbf{B}$ is not computed self-consistently with the sheaths.) Completing the circuit with a path in the metal frame of the antenna and using Faraday's Law, one can show that this integral equals the magnetic flux through the circuit. ${ }^{7}$ Thus, this integral represents an external electromagnetic (EM) drive for the sheath voltage. The general feature of the SBC and the model problem that should survive in the EM case is that (for $\Lambda$ >>1) the plasma screens $\mathrm{E}_{\|}^{\mathrm{vac}}$ which then appears in the sheaths such that $\int \mathrm{ds} \mathrm{E}_{\|}^{\mathrm{vac}}=\sum \Delta \mathrm{E}_{\|}^{\text {sh }}$ where the summation is over the two sheaths at each end of the field line.

For monopole (0-0) phasing in two-strap antennas $\int \mathrm{ds} \mathrm{E}_{\|}^{\mathrm{vac}} \neq 0$ and parity requires $E_{\|}^{\text {sh }}$ to be the same in each sheath, resulting in finite (typically large) sheath voltages. Note that the redistribution of $\mathrm{E}_{\|}$in the self-consistent rf field-sheath solution to satisfy the sheath BC (as illustrated in Fig. 5) does not change the magnetic flux through the circuit. Thus, we expect the new approach to yield similar results to the older, approximate method of estimating electromagnetically-driven sheath voltages when $\Lambda$ >> 1. Conversely, when $\Lambda<<1$, as happens with sufficiently long systems $(L \rightarrow \infty)$, the screening is incomplete, and we speculate that $\int \mathrm{ds}_{\|}^{\mathrm{vac}}$ significantly overestimates the sheath voltage. [See the discussion following Eq. (32).] 
For dipole $(0-\pi)$ phasing $\int \mathrm{ds}_{\|}^{\mathrm{vac}} \approx 0$ due to cancellation of the magnetic flux from adjacent current straps. This requirement can be satisfied in two ways. Either $E_{\|}^{\text {sh }}$ $=0$ for both sheaths or $E_{\|}^{\text {sh }}$ has opposite signs in the two sheaths. The later case is the parity of the EPW mode considered in this paper. (Our EPW is, of course, driven directly by launching it down a waveguide.) We speculate that dipole ICRF antennas have very small coupling to this EPW mode, and consequently give rise to very small sheaths, consistent with experimental observations.

Motivated by the success of our model electrostatic calculation, the next step will be implementation of the sheath $\mathrm{BC}$ in antenna and wave propagation codes including electromagnetic physics. The sheath BC [Eq. (9)] is relevant to both fast- and slow-wave propagation and should be included in calculations of both near- and far-field effects. Applications of the sheath $\mathrm{BC}$ to fast wave propagation will be important in optimizing ICRF heating in ITER and other future machines. Work is in progress to incorporate this boundary condition in codes that include the fast wave polarization and will be reported elsewhere.

\section{Acknowledgements}

We would like to thank M. D. Carter, L. A. Berry, E. F. Jaeger, and D. Smithe for stimulating discussions of sheath BCs and for collaboration on related problems as part of the rf SciDAC project. We would also like to thank P. T. Bonoli and J. C. Wright for discussions of lower hybrid physics. This work was supported by the U.S. Department of Energy (DOE) under DOE Grants No. DE-FG02-97ER54392 and DE-FC02-05ER54823; however, this support does not constitute an endorsement by the DOE of the views expressed herein. 


\section{Appendix A: Energy Conservation}

In this Appendix, we demonstrate that the self-consistent EPW solution obtained from the sheath BC satisfies energy conservation in the limit $v<<1$ but for arbitrary $\eta$. In this derivation, we use the geometry of our model problem, so that $\mathrm{Q}_{\perp}=\mathrm{Q}_{\mathrm{X}}$ and $\mathrm{Q}_{\|}=\mathrm{Q}_{\mathrm{Z}}$ for any vector $\mathbf{Q}$ and $\varepsilon_{\|} \equiv \varepsilon_{\mathrm{zz}}$ and $\varepsilon_{\perp} \equiv \varepsilon_{\mathrm{Xx}}$ for the tensor $\boldsymbol{\varepsilon}$.

The starting point is the steady-state version of Poynting's Theorem

$$
\nabla \cdot \mathbf{S}+\mathbf{J} \cdot \mathbf{E}=0 \Rightarrow \frac{\partial}{\partial x} \int \mathrm{dz} \mathrm{S}_{\mathrm{x}}=-\mathrm{P}
$$

where $\mathbf{S}=($ c $/ 4 \pi)<\mathbf{E} \times \mathbf{B}>$, the time average is defined by $<\mathbf{A B}>=\left(\mathbf{A}^{*} \mathbf{B}+\right.$ c.c. $) / 4$, and $\mathrm{P}=\mathrm{P}_{\mathrm{sh}} / \mathrm{A}$ denotes the power per unit area.

For the slow wave (SW) polarization and with $\mathrm{k}_{\mathrm{y}}=0$, the Poynting flux $\mathrm{S}_{\mathrm{x}}$ and the power dissipation $\mathrm{P}$ are defined by

$$
\mathrm{S}_{\mathrm{x}} \equiv-\frac{\mathrm{c}}{16 \pi} \mathrm{E}_{\mathrm{z}}^{*} \mathrm{~B}_{\mathrm{y}}+\mathrm{cc}, \quad \mathrm{P} \equiv \int \mathrm{dz} \frac{1}{4} \mathrm{~J}_{\mathrm{z}} \mathrm{E}_{\mathrm{z}}^{*}+\mathrm{Cc}
$$

The rf field $B_{y}$ is obtained from the $z$ component of Ampere's Law using Eq. (11) for $\mathrm{J}_{\mathrm{z}}$ :

$$
\mathrm{ik}_{\mathrm{x}} \mathrm{B}_{\mathrm{y}} \equiv \frac{4 \pi}{\mathrm{c}} \mathrm{J}_{\mathrm{z}}-\frac{\mathrm{i} \omega}{\mathrm{c}} \mathrm{E}_{\mathrm{z}}=-\frac{\mathrm{i} \omega}{\mathrm{c}} \varepsilon_{\mathrm{zz}} \mathrm{E}_{\mathrm{z}} \Rightarrow \mathrm{B}_{\mathrm{y}} \equiv-\frac{\omega}{\mathrm{k}_{\mathrm{x}} \mathrm{c}} \varepsilon_{\mathrm{zz}} \mathrm{E}_{\mathrm{z}},
$$

Combining Eqs. (A2) and (A3) yields the Poynting flux

$$
\mathrm{S}_{\mathrm{x}}=\frac{\omega}{8 \pi \mathrm{k}_{\mathrm{x}}} \varepsilon_{\|}\left|\mathrm{E}_{\mathrm{z}}\right|^{2}
$$

Expressing $\mathrm{E}_{\mathrm{z}}=-\mathrm{i} \mathrm{k}_{\mathrm{z}} \Phi$, with $\Phi$ defined in Eq. (20), we obtain the local Poynting flux in the plasma region and its integrated value

$$
\mathrm{S}_{\mathrm{x}}^{(\mathrm{pl})}=\frac{\omega}{8 \pi \mathrm{k}_{\mathrm{x}}} \varepsilon_{\|} \mathrm{k}_{\mathrm{z}}{ }^{2} \Phi_{0}{ }^{2} \sin ^{2} \mathrm{k}_{\mathrm{z}} \mathrm{z}, \int_{-\mathrm{L}}^{\mathrm{L}} \mathrm{dz} \mathrm{S}_{\mathrm{x}}^{(\mathrm{pl})}=\frac{\omega \mathrm{k}_{\mathrm{z}} \varepsilon_{\|}}{16 \pi \mathrm{k}_{\mathrm{x}}} \Phi_{0}{ }^{2}(2 \eta-\sin 2 \eta)
$$

In the sheath region, $\mathrm{E}_{\mathrm{z}}^{(\mathrm{sh})}=\mathrm{V}_{\mathrm{sh}} / \Delta=\varepsilon_{\mathrm{zz}}^{(\mathrm{pl})} \mathrm{E}_{\mathrm{z}}^{(\mathrm{pl})}$ using the continuity of $\mathrm{D}_{\mathrm{z}}$ across the sheath-plasma interface and the fact that $\varepsilon_{\mathrm{zz}}=1$ in the sheath region. Using this result 
with $\mathrm{V}_{\text {sh }}$ given by Eq. (32), we obtain the local Poynting flux in the sheath region and its value integrated over both sheaths

$$
\mathrm{S}_{\mathrm{x}}^{(\mathrm{sh})}=\frac{\omega}{8 \pi \mathrm{k}_{\mathrm{x}} \Delta^{2}} \Phi_{0}{ }^{2} \cos ^{2} \eta, \quad \int_{(2 \Delta)} \mathrm{dz} \mathrm{S}_{\mathrm{x}}^{(\mathrm{sh})}=\frac{\omega}{4 \pi \mathrm{k}_{\mathrm{x}} \Delta} \Phi_{0}{ }^{2} \cos ^{2} \eta \quad .
$$

Combining the results in Eqs. (A5) and (A6) gives the total Poynting flux, integrated over all z. We use the definition of $\Lambda$ in Eq. (28) and the identity (29) to eliminate $\Delta$; after some algebra one can obtain the simple form for the total Poynting flux

$$
\int_{-\mathrm{L}}^{\mathrm{L}} \mathrm{dz} S_{\mathrm{x}}=\frac{\omega \varepsilon_{\|}}{16 \pi} \frac{\mathrm{k}_{\mathrm{z}}}{\mathrm{k}_{\mathrm{x}}} \Phi_{0}^{2}(2 \eta+\sin 2 \eta)
$$

For the EPW, power dissipation occurs only in the sheaths. The current is given by Eq. (11), $E_{\mathrm{z}}^{(\mathrm{sh})}=\mathrm{V}_{\mathrm{sh}} / \Delta$, and $\mathrm{P}$ is defined by the second relation in Eq. (A2). Integrating over both sheaths $\left(\int \mathrm{dz} \rightarrow 2 \Delta\right)$ we obtain

$$
\mathrm{P} \equiv \int \mathrm{dz} \frac{\omega \mathrm{V}}{8 \pi}\left|\mathrm{E}_{\mathrm{z}}\right|^{2}=\frac{\omega \mathrm{V}}{4 \pi \Delta} \Phi_{0}^{2} \cos ^{2} \eta
$$

Using Eq. (28) to eliminate $\Delta$ gives

$$
\mathrm{P}=-\frac{\omega \nu}{4 \pi} \frac{\varepsilon_{\|}}{\Lambda \mathrm{L}} \cos ^{2} \eta \Phi_{0}^{2}
$$

Combining the results for $\mathrm{P}$ and $\int \mathrm{dz} \mathrm{S}_{\mathrm{X}}$, using the fact that the dielectric tensor and $\mathrm{k}_{\mathrm{Z}} / \mathrm{k}_{\mathrm{x}}$ are constant in space, and using the identity in Eq. (29), we recover the nonlinear mode equation, Eq. (41), viz.

$$
\nabla_{\mathrm{x}}\left((\sin 2 \eta+2 \eta) \Phi_{0}^{2}\right)=-\frac{2 v}{\mathrm{~L}} \frac{\mathrm{k}_{\mathrm{x}}}{\mathrm{k}_{\mathrm{z}}} \eta \sin 2 \eta \Phi_{0}^{2}
$$

This proves that our calculation using the sheath BC satisfies energy conservation, validating the two-scale method and the $v$ expansion. We expect that this property will 
also hold in the numerical implementation of the BC in more general settings, where one iterates the nonlinear sheath relations and the wave fields to convergence.

\section{Appendix B: High Frequency Limit}

As discussed in the main body of the paper, the present work is based on sheath theory 6,21,22 that assumes (i) immobile ions and (ii) electrons without inertia which move primarily along field lines. This restricts the theory to the frequency range

$$
\omega_{\mathrm{pi}} \sin \alpha<<\omega<<\min \left(\omega_{\mathrm{pe}}, \mathrm{v}_{\mathrm{e}} / \Delta\right) \sin \alpha
$$

where $\alpha=B_{n} / B$ is the equilibrium magnetic field line angle with respect to the sheath.

The question naturally arises about what happens in the high frequency limit, e.g. whether the theory applies in the lower hybrid frequency regime and how it scales with frequency. We restrict the present discussion to the low density region around the antenna or waveguide coupler, where $\varepsilon_{\perp}=1$ and $\omega_{\text {pe }}$ can be small. In the LH frequency range, the right hand inequality in Eq. (B1) is not always satisfied, i.e. the effects of electron mass must be retained in the sheath theory.

To understand what happens at high frequencies, we consider the limit $\omega \rightarrow \infty$, for which the electrons are not be able to respond to the rf fields and act as if they are immobile. In this limit, there is no increased electron current to the wall (in response to the rf fields) to drive the rf sheath, and the rectification effect disappears. Thus, we expect $\mathrm{C}_{\mathrm{sh}}$ to be a decreasing function of frequency when electron mass effects are retained in the theory. The frequency scaling of the sheath width can be inferred from Eq. (13), $\Delta \propto$ $\mathrm{C}_{\mathrm{sh}}{ }^{3 / 4}$, and from Eq. (16) we find that $\mathrm{P}_{\mathrm{sh}} \propto \mathrm{C}_{\mathrm{sh}}$. The sheath parameter $\Lambda$ defined in Eq. (28), $\Lambda=(\Delta / \mathrm{L})\left(\omega_{\mathrm{pe}} / \omega\right)^{2} \propto \mathrm{C}_{\mathrm{sh}}^{3 / 4 / \omega^{2}}$. Thus, $\Delta, \mathrm{P}_{\mathrm{sh}}$ and $\Lambda \rightarrow 0$ in the high frequency limit. It can be shown from Eq. (A7) that the wave amplitude $\Phi$ is constant (independent 
of $\omega$ ) at fixed rf power (taking $\varepsilon_{\perp} \sim 1$ ). Combining these results in the definition of the scale length for power loss in Eq. (50), we find that $\mathrm{L}_{\text {loss }} \sim$ const./ $\mathrm{C}_{\mathrm{sh}} \rightarrow \infty$ as $\omega \rightarrow \infty$.

Applying this discussion to the LH range of frequencies, we conclude that the present formalism is valid for LH waves if Eq. (B1) is satisfied, but the magnitude of the sheath effects (rectification, power loss, redistribution of fields) will be greatly reduced by the effect of electron inertia at higher frequencies [violating Eq. (B1)].

\section{References}

1 “Nonlinear ICRF-plasma interactions,” J. R. Myra, D. A. D’Ippolito, D.A. Russell, L.A. Berry, E.F. Jaeger and M.D. Carter, Nucl. Fusion 46, S455 (2006).

${ }^{2}$ H. S. Butler and G. S. Kino, Phys. Fluids 6, 1346 (1963).

${ }^{3}$ F. W. Perkins, Nucl. Fusion 29, 583 (1989).

${ }^{4}$ R. Chodura and J. Neuhauser, in Proceedings of the $16^{\text {th }}$ European Conference on Controlled Fusion and Plasma Heating, Venice (European Physical Society, Petit-Lancy, 1989) Vol. 13B, Part III, p. 1089; R. Chodura, Fusion Eng. Design 12, 111 (1990).

${ }^{5}$ R. Van Niewenhove and G. Van Oost, J. Nucl. Mat. 162-164, 288 (1989).

${ }^{6}$ J. R. Myra, D. A. D’Ippolito, and M. J. Gerver, Nucl. Fusion 30, 845 (1990).

${ }^{7}$ D. A. D’Ippolito, J. R. Myra, M. Bures, and J. Jacquinot, Plasma Phys. Controlled Fusion 33, 607 (1991).

${ }^{8}$ R. Van Nieuwenhove, G. Van Oost, P. E. Vandenplas, R. A. Moyer, D. Gray, and R. W. Conn, Fusion Eng. Design 12, 231 (1990).

${ }^{9}$ D. A. D’Ippolito, J. R. Myra, J. Jacquinot , and M. Bures, Phys. Fluids B 5, 3603 (1993).

${ }^{10}$ M. Bécoulet, L. Colas, S. Pécoul, J. Gunn, Ph. Ghendrih, A. Bécoulet, and S. Heuraux, Phys. Plasmas 9 , 2619 (2002).

11 J. R. Myra, D. A. D’Ippolito, and Y. L. Ho, Fusion Eng. Design 31, 291 (1996).

${ }^{12}$ L. Colas, L. Costanzo, C. Desgranges, et al., Nucl. Fusion 43, 1 (2003).

${ }^{13}$ D. A. D’Ippolito and J. R. Myra, Phys. Plasmas 3, 420 (1996).

14 J. R. Myra, D. A. D’Ippolito, and M. Bures, Phys. Plasmas 1, 2890 (1994).

${ }^{15}$ L. Colas, E. Faudot, S. Brémond, et al., in Proceedings of 16th Topical Conference on Radio Frequency Power in Plasmas, (AIP, New York, 2005), p. 150; L. Colas, E. Faudot, S. Brémond, et al., Nucl. Fusion 46, S500 (2006).

${ }^{16}$ D. A. D’Ippolito, J. R. Myra, D. A. Russell, and M. D. Carter, in Proceedings of 16th Topical Conference on Radio Frequency Power in Plasmas, (AIP, New York, 2005), p. 222.

17 J. R. Myra, D. A. D’Ippolito, D. A. Russell, J. H. Rogers, and T. Intrator, Phys. Plasmas 7, 283 (2000). 
18 T. Intrator, J. R. Myra and D. A. D’Ippolito, Nucl. Fusion 43, 531 (2003).

${ }^{19}$ D. F. H. Start, G. Bell, V. P. Bhatnagar, et al., in Proceedings of 11th Topical Conference on Radio Frequency Power in Plasmas, (AIP, New York, 1995), p. 7.

${ }^{20}$ J. C. Hosea, S. Bernabei, T. Biewer, B. LeBlanc, C. K. Phillips, J. R. Wilson, D. Stutman, P. Ryan, and D. W. Swain, in Proceedings of 16th Topical Conference on Radio Frequency Power in Plasmas, (AIP, New York, 2005), p. 82.

${ }^{21}$ M. A. Lieberman, IEEE Trans. Plasma Sci. PS-16, 638 (1988).

${ }^{22}$ V. A. Godyak and N. Sternberg, Phys. Rev. A 42, 2299 (1990).

${ }^{23}$ E. F. Jaeger, L. A. Berry, J. S. Tolliver, and D. B. Batchelor, Phys. Plasmas 2, 2597 (1995).

${ }^{24}$ M. D. Carter, V. Godyak, D. Hoffman, W.S. Lee, D. Buchberger, and P. M. Ryan, submitted to J. Appl. Phys. 99 (2006).

${ }^{25}$ D. Smithe, private communication (2005).

${ }^{26}$ R. Majeski, P. H. Probert, T. Tanaka, et al., Fusion Eng. Design 24, 159 (1994).

27 J. Sorensen, D. Diebold, R. Majeski, N, Hershkowitz, Nucl. Fusion 36, 173 (1996).

${ }^{28}$ J. Menard, W. Choe, R. Majeski, M. Ono, D. Stutman, J.R. Wilson, and T. Seki, Bull. Am. Phys. Soc. 41, 1402 (1996), paper 2R 16.

29 D. A. D’Ippolito, J. R. Myra, P. M. Ryan, E. Righi, J. Heikkinen, P. Lamalle, J.-M. Noterdaeme, and Contributors to the EFDA-JET Workprogramme, Nucl. Fusion 42, 1356 (2002).

${ }^{30}$ J. R. Myra, D. A. D’Ippolito, D. W. Forslund and J. U. Brackbill, Phys. Rev. Lett. 66, 1173 (1991).

31 J. R. Myra, D. A. D’Ippolito, X.Q. Xu, and R.H. Cohen, Contrib. Plasma Phys. 40, 352 (2000). 


\section{Figure Captions}

Fig. 1 Schematic of the general sheath problem and the two coordinate systems defined in the text. Shown are the unit vectors $\left(\mathbf{n}, \mathbf{e}_{t}\right)$ normal and tangential to the sheathplasma interface and (b, $\left.\mathbf{e}_{\perp}\right)$ along and perpendicular to the magnetic field $\mathbf{B}$. The normal $\mathbf{n}$ is defined to point from the sheath into the plasma region.

Fig. 2 Schematic of the model SW sheath problem. The unit normal $\mathbf{n}$ at the sheathplasma interface is defined to point from the sheath into the plasma region, and the magnetic field $\mathbf{B}$ points to the right. With these definitions, $\mathbf{n} \cdot \mathbf{b}$ is +1 at the left-hand sheath and -1 at the right-hand sheath.

Fig. 3 Sketch of lowest order eigenmode. Note that $\varepsilon$ changes sign in going from the plasma to the sheath region, so in order for $D_{z}=\varepsilon E_{z}$ to match, $E_{z}$ must also change sign across the $\mathrm{z}=\mathrm{L}$ interface. Thus we expect the lowest order even modes to have $\mathrm{k}_{\mathrm{z}} \mathrm{L}$ in the range $\pi / 2<\mathrm{k}_{\mathrm{z}} \mathrm{L}<\pi$.

Fig. 4 Plot of the numerical solution of Eq. (44) for the normalized potential $\Phi(\mathrm{x})$ (solid curve), the normalized rectified potential $\mathrm{V}_{0}(\mathrm{x})=\mathrm{C}_{\mathrm{sh}} \mathrm{V}_{\mathrm{sh}}=\mathrm{C}_{\mathrm{sh}} \Phi|\cos \eta|$ (longdashed curve) and the ratio $\mathrm{R}_{\mathrm{P}}$ of the integrated sheath power dissipation to initial Poynting flux (short-dashed curve), as defined in Eq. (49), for the case $\Phi_{\max }=2$. Here, $\Phi$ and $\mathrm{V}_{0}$ are normalized to $\Phi_{\Lambda}$ and $\mathrm{x}$ is normalized to the nonlinear scale length $\mathrm{L}_{\mathrm{X}}=-\mathrm{L} \mathrm{k}_{\mathrm{Z}} / \mathrm{k}_{\mathrm{X}} \mathrm{v}_{0}$. 
Fig. 5 Plot of $\mathrm{E}_{\mathrm{z}}^{(\mathrm{pl})} / \mathrm{E}_{\mathrm{z} 0}^{(\mathrm{pl})} \mathrm{vs} \Phi$ with $\mathrm{E}_{\mathrm{Z}}$ evaluated at the sheath $(\mathrm{z}=\mathrm{L})$ and $\Phi$ normalized to $\Phi_{\Lambda}$. As explained in the text, this compares the $\mathrm{E}_{\mathrm{Z}}$ fields obtained with the sheath $\mathrm{BC}(\Lambda \neq 0)$ and the metal wall $\mathrm{BC}(\Lambda=0)$. 


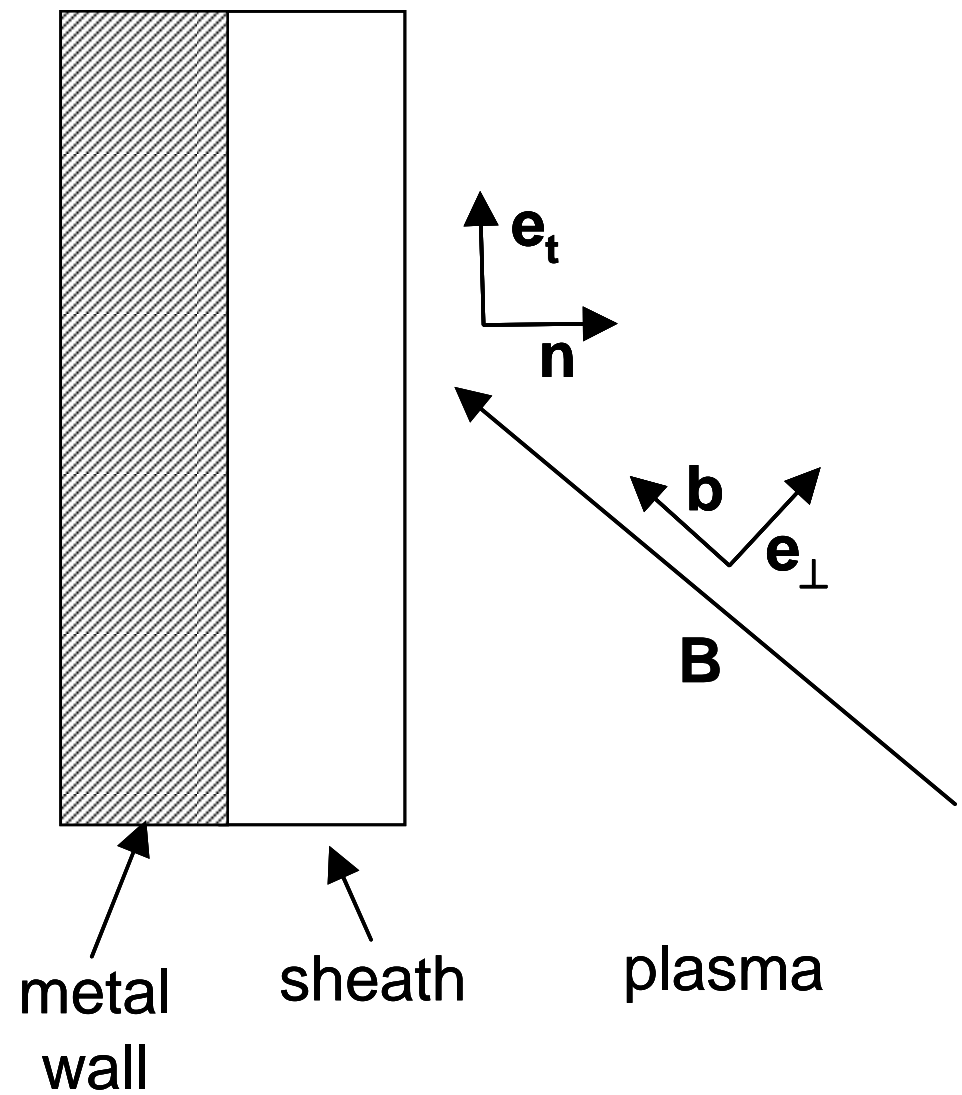

Fig. 1 Schematic of the general sheath problem and the two coordinate systems defined in the text. Shown are the unit vectors $\left(\mathbf{n}, \mathbf{e}_{\mathrm{t}}\right)$ normal and tangential to the sheath-plasma interface and $\left(\mathbf{b}, \mathbf{e}_{\perp}\right)$ along and perpendicular to the magnetic field $B$. The normal $\mathbf{n}$ is defined to point from the sheath into the plasma region. 


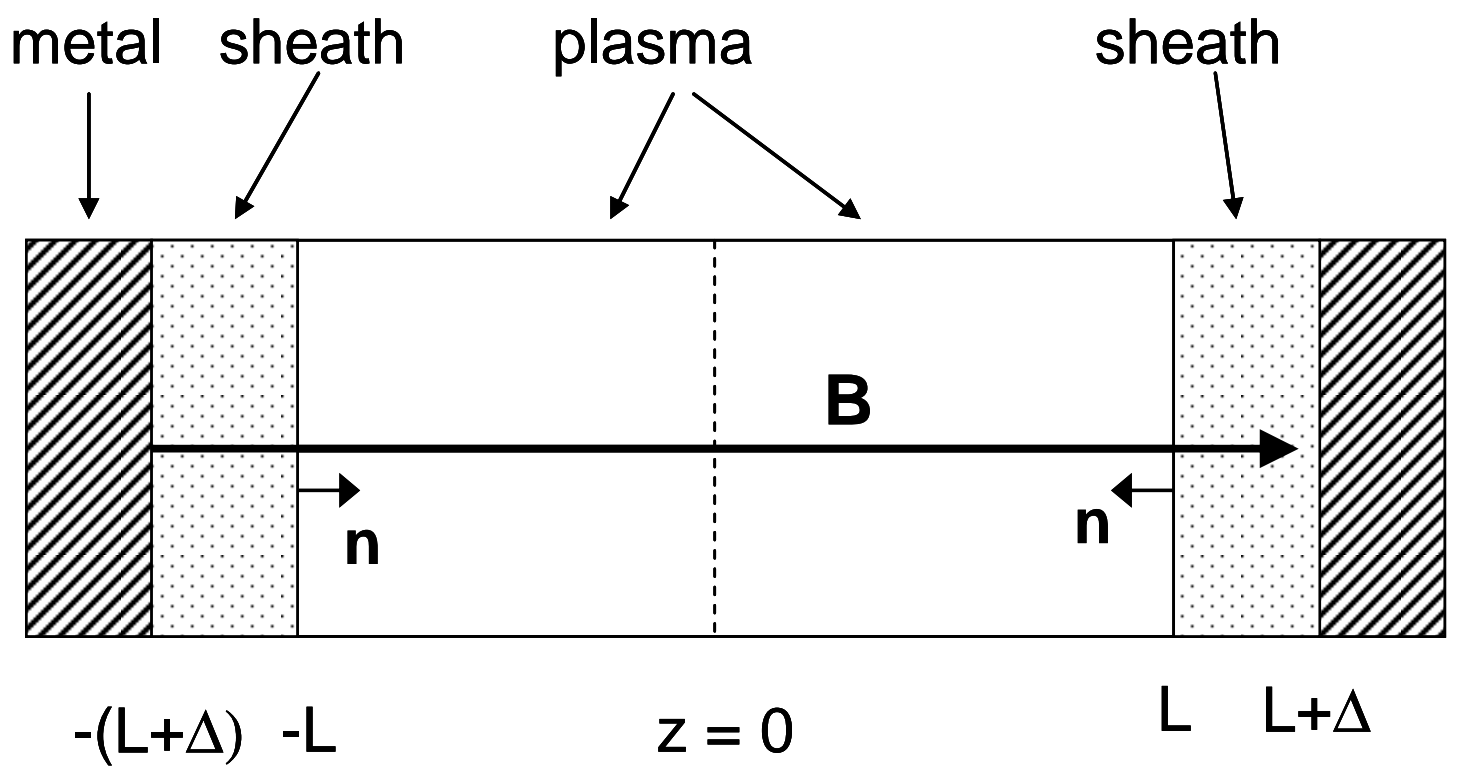

Fig. 2 Schematic of the model SW sheath problem. The unit normal $\mathbf{n}$ at the sheath-plasma interface is defined to point from the sheath into the plasma region, and the magnetic field $\mathbf{B}$ points to the right. With these definitions, $\mathbf{n} \cdot \mathbf{b}$ is +1 at the left-hand sheath and -1 at the right-hand sheath. 


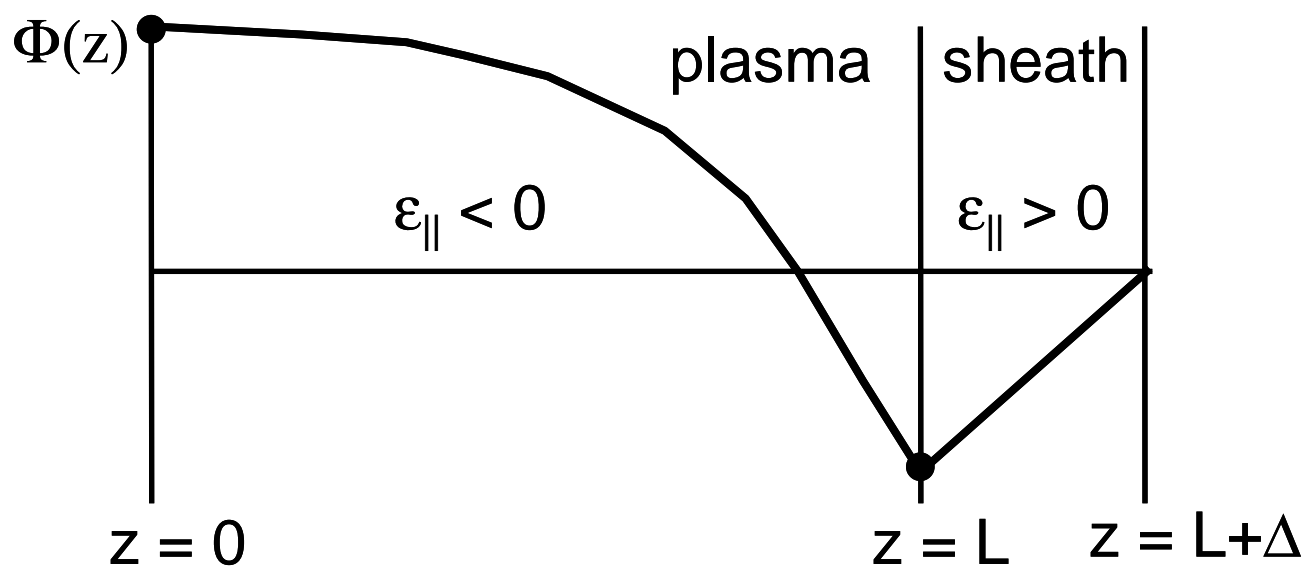

Fig. 3 Lowest order eigenmode. Note that $\varepsilon$ changes sign in going from the plasma to the sheath region, so in order for $D_{Z}=\varepsilon E_{Z}$ to match, $E_{Z}$ must also change sign across the $z=L$ interface. Thus we expect the lowest order even modes to have $k_{z} L$ in the range $\pi / 2<k_{Z} L<\pi$. 


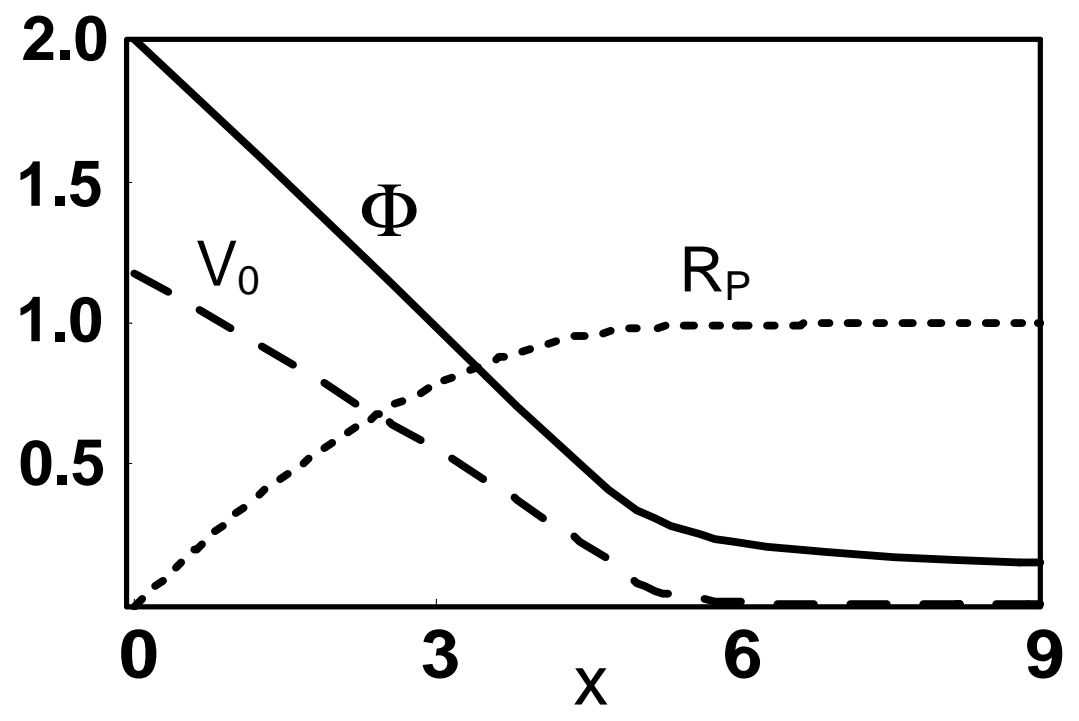

Fig. 4 Plot of the numerical solution of Eq. (44) for the normalized potential $\Phi(x)$ (solid curve), the normalized rectified potential $V_{0}$ (long-dashed curve) and the ratio $R_{P}$ of the integrated sheath power dissipation to initial Poynting flux (shortdashed curve), as defined in Eq. (49), for the case $\Phi_{\max }=2$. Here, $\Phi$ and $V_{0}$ are normalized to $\Phi_{\Lambda}$ and $x$ is normalized to the nonlinear scale length $L_{X}=-$ $\mathrm{Lk}_{\mathrm{Z}} / \mathrm{k}_{\mathrm{X}} \mathrm{v}_{0}$. 


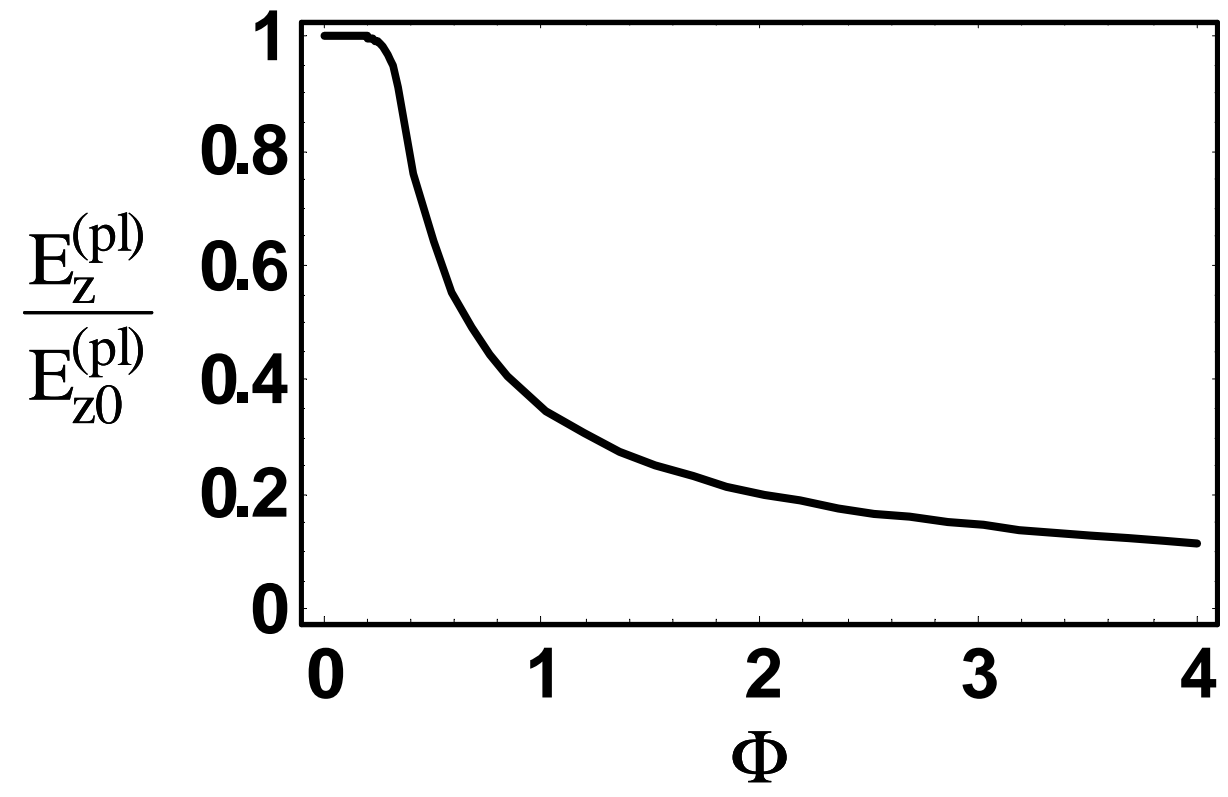

Fig. 5 Plot of $\mathrm{E}_{\mathrm{z}}^{(\mathrm{pl})} / \mathrm{E}_{\mathrm{z} 0}^{(\mathrm{pl})}$ vs $\Phi$ with $\mathrm{E}_{\mathrm{Z}}$ evaluated at the sheath $(\mathrm{z}=\mathrm{L})$ and $\Phi$ normalized to $\Phi_{\Lambda}$. As explained in the text, this compares the $E_{Z}$ fields obtained with the sheath $\mathrm{BC}(\Lambda \neq 0)$ and the metal wall $\mathrm{BC}(\Lambda=0)$. 